ALEA, Lat. Am. J. Probab. Math. Stat. 15, 1215-1239 (2018)

DOI: $10.30757 /$ ALEA.v15-45

\title{
Prediction of weakly locally stationary processes by auto-regression
}

\author{
François Roueff and Andrés Sánchez-Pérez \\ LTCI, Télécom ParisTech, Université Paris-Saclay \\ 46, rue Barrault, \\ 75634 Paris Cedex 13, France. \\ E-mail address: francois.roueff@telecom-paristech.fr \\ URL: https://perso.telecom-paristech.fr/ roueff/ \\ LTCI, Télécom ParisTech, Université Paris-Saclay \\ 46, rue Barrault, \\ 75634 Paris Cedex 13, France. \\ E-mail address: andres.sanchez-perez@polytechnique.edu
}

\begin{abstract}
In this contribution we introduce weakly locally stationary time series through the local approximation of the non-stationary covariance structure by a stationary one. This allows us to define autoregression coefficients in a non-stationary context, which, in the particular case of a locally stationary Time Varying Autoregressive (TVAR) process, coincide with the generating coefficients. We provide and study an estimator of the time varying autoregression coefficients in a general setting. The proposed estimator of these coefficients enjoys an optimal minimax convergence rate under limited smoothness conditions. In a second step, using a bias reduction technique, we derive a minimax-rate estimator for arbitrarily smooth time-evolving coefficients, which outperforms the previous one for large data sets. In turn, for TVAR processes, the predictor derived from the estimator exhibits an optimal minimax prediction rate.
\end{abstract}

\section{Introduction}

In many applications, one is interested in predicting the next values of an observed time series. It is the case in various areas like finance (stock market, volatility on prices), social sciences (population studies), epidemiology, meteorology and

Received by the editors 2017-01-24; accepted 2018-10-01.

2010 Mathematics Subject Classification. 62M20, 62G99, 62M10, 68W27.

Key words and phrases. Locally stationary time series, auto-regression coefficients, time varying autoregressive processes, minimax-rate prediction.

This work has been partially supported by the Conseil régional d'Île-de-France under a doctoral allowance of its program Réseau de Recherche Doctoral en Mathématiques de l'Île de France (RDM-IdF) for the period 2012 - 2015 and by the Labex LMH (ANR-11-IDEX-003-02). 
network systems (Internet traffic). Autoregressive processes have been used successfully in a stationary context for several decades. On the other hand, in a context where the number of observations can be very large, the usual stationarity assumption has to be weakened to take into account some smooth evolution of the environment.

Several prediction methods developed in signal processing are well known to adapt to a changing environment. This is the case of the wide spread recursive least square algorithms. The initial goal of these methods is to provide an online procedure for estimating a regression vector with low numerical cost. Such methods usually rely on a forgetting factor or a gradient step size $\gamma$ and they can be shown to be consistent in a stationary environment when $\gamma$ decreases adequately to zero (see e.g. Duflo, 1997). Even when the environment is changing, that is, when the regression parameter evolves along the time, a "small enough" $\gamma$ often yields a good tracking of the evolving regression parameter. In order to have a sound and comprehensive understanding of this phenomenon, an interesting approach is to consider a local stationarity assumption, as successfully initiated in Dahlhaus (1996b) by relying on a non-stationary spectral representation introduced in Priestley (1965); see also Subba Rao et al. (2012) and the references therein for a recent overview. The basic idea is to provide an asymptotic analysis for the statistical inference of non-stationary time series such as time varying autoregressive (TVAR) processes using local stationary approximations. The analysis of the Normalized Least Mean Squares (NLMS) algorithm for tracking a changing autoregression parameter in this framework is tackled in Moulines et al. (2005). Such an analysis is based on the usual tools of non-parametric statistics. The TVAR parameter $\boldsymbol{\theta}$ is seen as the regular samples of a smooth $\mathbb{R}^{d}$-valued function. An in-fill asymptotic allows one to derive the rates of convergence of the NLMS estimator for estimating this function within particular smoothness classes of functions. As shown in Moulines et al. (2005), it turns out that the NLMS algorithm provides an optimal minimax rate estimator of the TVAR parameter with Hölder smoothness index $\beta \in(0,1]$. However it is no longer optimal for $\beta>1$, that is, when the TVAR parameter is smoother than a continuously differentiable function. An improvement of the NLMS is proposed in Moulines et al. (2005) to cope with the case $\beta \in(0,2]$ but, to the best of our knowledge, there is no available method neither for the $\boldsymbol{\theta}$ minimaxrate estimation nor for the minimax-rate prediction when $\beta>2$, that is when the TVAR parameter is smoother than a two-times continuously differentiable function.

In the present work, our main contribution is twofold. First we extend the concept of time-varying linear prediction coefficients to a general class of weakly locally stationary processes, which includes the class of locally stationary processes as introduced in Dahlhaus (1996b). In the specific case of a TVAR process, these coefficients correspond to the time-varying autoregression parameters. Second, we show that the tapered Yule-Walker estimator introduced in Dahlhaus and Giraitis (1998) for TVAR processes also applies to this general class and is minimax-rate for Hölder indices up to $\beta=1$ for asymmetric tapers and up to $\beta=2$ for symmetric ones. Moreover, by applying a bias reduction technique, we derive a new estimator which is minimax-rate for any arbitrarily large Hölder index $\beta$. By achieving this goal, we provide a theoretically justified construction of predictors that can be chosen optimally, depending on how smoothly the time varying spectral density evolves along the time. On the other hand, in practical situations, one may not 
have a clear view on the value of the smoothness index $\beta$ and one should rely on data driven methods that are therefore called adaptive. This problem was recently tackled in Giraud et al. (2015) . More precisely, using aggregation techniques introduced in the context of individual sequences prediction (see e.g. Cesa-Bianchi and Lugosi, 2006) and statistical learning (see e.g. Barron, 1987), one can aggregate sufficiently many predictors in order to build a minimax predictor which adapts to the unknown smoothness $\beta$ of the time varying parameter of a TVAR process. However, a crucial requirement in Giraud et al. (2015) is to rely on $\beta$-minimaxrate sequences of predictors for any $\beta>0$. Our main contribution here is to fill this gap, hence achieving to solve the problem of the adaptive minimax-rate linear forecasting of locally stationary TVAR processes with coefficients of any (unknown, arbitrarily large) Hölder smoothness index.

The paper is organized as follows. In Section 2, we introduce a definition of weakly locally stationary time series, the regression problem investigated in this work in relation with the practical prediction problem, and the tapered Yule-Walker estimator under study. General results on this estimator are presented in Section 3 and a minimax rate estimator is derived. The particular case of TVAR processes is treated in Section 4. Numerical experiments illustrating these results can be found in Section 5. Postponed proofs and useful lemmas are provided in the appendices.

\section{General setting}

In the following, non-random vectors and sequences are denoted using boldface symbols, $\|\mathbf{x}\|$ denotes the Euclidean norm of $\mathbf{x},\|\mathbf{x}\|=\left(\sum_{i}\left|x_{i}\right|^{2}\right)^{1 / 2}$, and $\|\mathbf{x}\|_{1}$ its $\ell_{1}$ norm, $\|\mathbf{x}\|_{1}=\sum_{i}\left|x_{i}\right|$. If $f$ is a function, $\|f\|_{\infty}=\sup _{x}|f(x)|$ corresponds to its sup norm. If $A$ is a matrix, $\|A\|$ denotes its spectral norm, $\|A\|=\sup \{\|A \mathbf{x}\|,\|\mathbf{x}\| \leq 1\}$. We moreover denote

$$
\ell^{1}(\mathbb{N})=\left\{\boldsymbol{x} \in \mathbb{R}^{\mathbb{N}} \text { s.t. }\|\boldsymbol{x}\|_{1}<\infty\right\} \quad \text { and } \quad \ell_{+}^{1}(\mathbb{N})=\left\{\boldsymbol{x} \in \mathbb{R}_{+}^{\mathbb{N}} \text { s.t. }\|\boldsymbol{x}\|_{1}<\infty\right\} .
$$

2.1. Main definitions. We consider a doubly indexed time series $\left(X_{t, T}\right)_{t \in \mathbb{Z}, T \in \mathbb{N}^{*}}$. Here $t$ refers to a discrete time index and $T$ is an additional index indicating the sharpness of the local approximation of the time series $\left(X_{t, T}\right)_{t \in \mathbb{Z}}$ by a stationary one. Coarsely speaking, $\left(X_{t, T}\right)_{t \in \mathbb{Z}, T \in \mathbb{N}^{*}}$ is considered to be weakly locally stationary if, for $T$ large, given a set $S_{T}$ of sample indices such that $t / T \approx u$ over $t \in S_{T}$, the sample $\left(X_{t, T}\right)_{t \in S_{T}}$ can be approximately viewed as the sample of a weakly stationary time series depending on the rescaled location $u$. Note that $u$ is a continuous time parameter, sometimes referred to as the rescaled time index. Following Dahlhaus (1996b), $T$ is usually interpreted as the number of available observations, in which case all the definitions are restricted to $1 \leq t \leq T$ and $u \in[0,1]$. However this is not essential in our mathematical derivations and it is more convenient to set $t \in \mathbb{Z}$ and $u \in \mathbb{R}$ for presenting our setting.

We use the following class of functions. For $\alpha \in(0,1]$ the $\alpha$-Hölder semi-norm of a function $\mathbf{f}: \mathbb{R} \rightarrow \mathbb{C}^{d}$ is defined by

$$
|\mathbf{f}|_{\alpha, 0}=\sup _{0<\left|s-s^{\prime}\right|<1} \frac{\left\|\mathbf{f}(s)-\mathbf{f}\left(s^{\prime}\right)\right\|}{\left|s-s^{\prime}\right|^{\alpha}} .
$$


This semi-norm is used to build a norm for any $\beta>0$ as it follows. Let $k \in \mathbb{N}$ and $\alpha \in(0,1]$ be such that $\beta=k+\alpha$. If $\mathbf{f}$ is $k$ times differentiable on $\mathbb{R}$, we define

$$
|\mathbf{f}|_{\beta}=\left|\mathbf{f}^{(k)}\right|_{\alpha, 0}+\max _{0 \leq s \leq k}\left\|\mathbf{f}^{(s)}\right\|_{\infty}
$$

and $|\mathbf{f}|_{\beta}=\infty$ otherwise. For $R>0$ and $\beta>0$, the $(\beta, R)-$ Hölder ball of dimension $d$ is denoted by

$$
\Lambda_{d}(\beta, R)=\left\{\mathbf{f}: \mathbb{R} \rightarrow \mathbb{C}^{d} \text {, such that }|\mathbf{f}|_{\beta} \leq R\right\}
$$

We first introduce definitions for the time varying covariance and the local covariance functions.

Definition 2.1 (Time varying covariance function). Let $\left(X_{t, T}\right)_{t \in \mathbb{Z}, T \in \mathbb{N}^{*}}$ be an array of random variables with finite variances. The local time varying covariance function $\gamma^{*}$ is defined for all $t \in \mathbb{Z}, T \in \mathbb{N}^{*}$ and $\ell \in \mathbb{Z}$ as

$$
\gamma^{*}(t, T, \ell)=\operatorname{cov}\left(X_{t, T}, X_{t-\ell, T}\right) .
$$

Definition 2.2 (Local covariance function and local spectral density). A local spectral density $f$ is a $\mathbb{R}^{2} \rightarrow \mathbb{R}_{+}$function, $(2 \pi)$-periodic and locally integrable with respect to the second variable. The local covariance function $\gamma$ associated with the local spectral density $f$ is defined on $\mathbb{R} \times \mathbb{Z}$ by

$$
\gamma(u, \ell)=\int_{-\pi}^{\pi} \mathrm{e}^{\mathrm{i} \ell \lambda} f(u, \lambda) \mathrm{d} \lambda .
$$

In (2.2), the variable $u$ should be seen as rescaled time index (in $\mathbb{R}$ ), $\ell$ as a (non-rescaled) time index and $\lambda$ as a frequency (in $[-\pi, \pi]$ ). Recall that, by the Herglotz theorem (see Brockwell and Davis, 2002, Theorem 4.3.1), Equation (2.2) guaranties that for any $u \in \mathbb{R},(\gamma(u, \ell))_{\ell \in \mathbb{Z}}$ is indeed the autocovariance function of a stationary time series. Now, we can state the definition of weakly locally stationary processes that we use here.

Definition 2.3 (Weakly locally stationary processes). Let $\left(X_{t, T}\right)_{t \in \mathbb{Z}, T \geq T_{0}}$ be an array of random variables with finite variances and $T_{0}, \beta, R>0$. We say that $\left(X_{t, T}\right)_{t \in \mathbb{Z}, T \geq T_{0}}$ is $(\beta, R)$-weakly locally stationary with local spectral density $f$ if, for all $\lambda \in \mathbb{R}$, we have $f(\cdot, \lambda) \in \Lambda_{1}(\beta, R)$, and the time varying covariance function $\gamma^{*}$ of $\left(X_{t, T}\right)_{t \in \mathbb{Z}, T \geq T_{0}}$ and the local covariance function $\gamma$ associated with $f$ satisfy, for all $t \in \mathbb{Z}$ and $\bar{T} \geq T_{0}$,

$$
\left|\gamma^{*}(t, T, \ell)-\gamma\left(\frac{t}{T}, \ell\right)\right| \leq R T^{-\min (1, \beta)} .
$$

Let us give some examples fulfilling this definition.

Example 2.4. Locally stationary processes were introduced in a general fashion by Dahlhaus (1996b) using the spectral representation

$$
X_{t, T}=\int_{-\pi}^{\pi} \mathrm{e}^{\mathrm{i} t \lambda} A_{t, T}^{0}(\lambda) \xi(\mathrm{d} \lambda)
$$

where $\xi(\mathrm{d} \lambda)$ is the spectral representation of a white noise and $\left(A_{t, T}^{0}\right)_{t \in \mathbb{Z}, T \in \mathbb{N}^{*}}$ is a collection of transfer functions such that there exist a constant $K$ and a (unique) 
$2 \pi$ - periodic function $A: \mathbb{R} \times \mathbb{R} \rightarrow \mathbb{C}$ with $A(u,-\lambda)=\overline{A(u, \lambda)}$ such that for all $T \geq 1$,

$$
\sup _{t \in \mathbb{Z}, \lambda \in \mathbb{R}}\left|A_{t, T}^{0}(\lambda)-A\left(\frac{t}{T}, \lambda\right)\right| \leq \frac{K}{T} .
$$

Provided adequate smoothness assumptions on the time varying transfer function $A$, this class of locally stationary processes satisfies Definition 2.3 (see Dahlhaus, 1996a, Section 1) for some $\beta \geq 1$ and $f(u, \lambda)=|A(u, \lambda)|^{2}$. The case $\beta \in(0,1]$ can be obtained by raising $T$ to the power $\beta$ in (2.5).

Example 2.5 (Time-varying Causal Bernoulli Shift (TVCBS)). Let $\varphi: \mathbb{R} \times \mathbb{R}^{\mathbb{N}} \rightarrow \mathbb{R}$. Consider, for all $T \geq 1$ and $t \in \mathbb{Z}$, a mapping $\varphi_{t, T}^{0}: \mathbb{R}^{\mathbb{N}} \rightarrow \mathbb{R}$ defining the random variables

$$
X_{t, T}=\varphi_{t, T}^{0}\left(\left(\xi_{t-k}\right)_{k \geq 0}\right),
$$

where $\left(\xi_{t}\right)_{t \in \mathbb{Z}}$ are i.i.d. We assume that $\mathbb{E}\left[\left|\xi_{0}\right|^{2 r}\right]<\infty$ for some $r \geq 1$ and that there exist $\beta, K>0$ and $\left(\psi_{k}\right)_{k \geq 0} \in \ell_{+}^{1}(\mathbb{N})$, such that, for all $T \geq 1, t \in \mathbb{Z}, u, u^{\prime} \in \mathbb{R}$ and $\boldsymbol{x} \in \mathbb{R}^{\mathbb{N}}$,

$$
\begin{aligned}
\left|\varphi_{t, T}^{0}(\boldsymbol{x})\right| & \leq K\left(1+\sum_{k=0}^{\infty} \psi_{k}\left|x_{k}\right|\right)^{r}, \\
\left|\varphi_{t, T}^{0}(\boldsymbol{x})-\varphi\left(\frac{t}{T}, \boldsymbol{x}\right)\right| & \leq K T^{-\min (1, \beta)}\left(1+\sum_{k=0}^{\infty} \psi_{k}\left|x_{k}\right|\right)^{r},
\end{aligned}
$$

It is easy to see that $\left(X_{t, T}\right)_{t \in \mathbb{Z}, T \geq T_{0}}$ satisfies (2.3) with a constant $R$ only depending on $K, \beta,\left(\psi_{k}\right)_{k \in \mathbb{N}}$ and $\mathbb{E}\left[\left|\xi_{0}\right|^{2 r}\right]$, and with local covariance function $\gamma(u, \cdot)$ defined as the covariance function of the stationary causal Bernoulli shift process $\left(X_{t}(u)\right)_{t \in \mathbb{Z}}$ defined by $X_{t}(u)=\varphi\left(u,\left(\left(\xi_{t-k}\right)_{k \geq 0}\right)\right.$. To obtain that $\left(X_{t, T}\right)_{t \in \mathbb{Z}, T \geq T_{0}}$ is $(\beta, R)$ weakly locally stationary, it thus only remains to check that $\left(X_{t}(u)\right)_{t \in \mathbb{Z}}$ admits a spectral density $f(u, \cdot)$ and that the resulting local spectral density satisfies $f(\cdot, \lambda) \in$ $\Lambda_{1}(\beta, R)$ for all $\lambda \in \mathbb{R}$.

Example 2.6 (TVAR $(p)$ model). Under suitable assumptions, the TVAR process is a particular case both of Example 2.4 (see Dahlhaus, 1996b, Theorem 2.3) and Example 2.5 (see Section 4). It is defined as the (unique) stable solution of the recursive equation

$$
X_{t, T}=\sum_{j=1}^{p} \theta_{j}\left(\frac{t}{T}\right) X_{t-j, T}+\sigma\left(\frac{t}{T}\right) \xi_{t},
$$

where $\boldsymbol{\theta}=\left[\theta_{1} \ldots \theta_{p}\right]^{\prime}: \mathbb{R} \rightarrow \mathbb{R}^{p}$ are the time varying autoregressive coefficients and $\left(\xi_{t}\right)_{t \in \mathbb{Z}}$ are i.i.d. centred and with variance 1. This example is detailed in Section 4.

2.2. Statement of the problem. Consider a weakly locally stationary $\left(X_{t, T}\right)_{t \in \mathbb{Z}, T \geq T_{0}}$, which we assume to have mean zero for convenience. Let $d \in \mathbb{N}^{*}$. For each $t=$ 
$1, \ldots, T$, define the prediction vector of order $d$ by

$$
\begin{aligned}
\boldsymbol{\theta}_{t, T}^{*} & =\underset{\boldsymbol{\theta}=\left[\theta_{1} \ldots \theta_{d}\right]^{\prime} \in \mathbb{R}^{d}}{\arg \min } \mathbb{E}\left[\left(X_{t, T}-\sum_{k=1}^{d} \theta_{k} X_{t-k, T}\right)^{2}\right] \\
& =\underset{\boldsymbol{\theta} \in \mathbb{R}^{d}}{\arg \min } \mathbb{E}\left[\left(X_{t, T}-\boldsymbol{\theta}^{\prime} \mathbf{X}_{t-1, T}\right)^{2}\right]
\end{aligned}
$$

where $A^{\prime}$ denotes the transpose of matrix $A$ and $\mathbf{X}_{s, T}=\left[\begin{array}{lll}X_{s, T} & \ldots X_{s-(d-1), T}\end{array}\right]^{\prime}$.

Let $\Gamma_{t, T}^{*}$ be the time varying covariances matrix $\Gamma_{t, T}^{*}=\left(\gamma^{*}(t-i, T, j-i) ; i, j=\right.$ $1, \ldots, d)$ where $\gamma^{*}$ is the time varying covariance function as defined in (2.1). Provided that $\Gamma_{t, T}^{*}$ is non-singular, the solution of (2.10) is given by

$$
\boldsymbol{\theta}_{t, T}^{*}=\left(\Gamma_{t, T}^{*}\right)^{-1} \gamma_{t, T}^{*}
$$

where $\boldsymbol{\gamma}_{t, T}^{*}=\left[\gamma^{*}(t, T, 1) \ldots \gamma^{*}(t, T, d)\right]^{\prime}$. Analogously to (2.11), and with the aim of approximating the local solution of the stationary Yule-Walker equations, we set

$$
\boldsymbol{\theta}_{u}=\Gamma_{u}^{-1} \gamma_{u},
$$

where $\gamma_{u}=\left[\begin{array}{llll}\gamma(u, 1) & \ldots & \gamma(u, d)\end{array}\right]^{\prime}, \Gamma_{u}$ is the covariances matrix $\Gamma_{u}=(\gamma(u, i-$ $j) ; i, j=1, \ldots, d)$ and $\gamma$ is the local covariance function as defined in (2.2). To ensure the above matrices to be non-singular, we assume a lower bound on the local spectral density, yielding the main assumption used on the model which depends on some positive constants $\beta, R$ and $f_{-}$and on a local spectral density $f$.

(M-1) The sequence $\left(X_{t, T}\right)_{t \in \mathbb{Z}, T \geq T_{0}}$ is a $(\beta, R)$-weakly locally stationary process with local spectral density $f$ in the sense of Definition 2.3. Assume moreover that the spectral density $f$ satisfies $f(u, \lambda) \geq f_{-}$for all $u, \lambda \in \mathbb{R}$.

The following lemma allows us to control the error of the approximation of the optimal linear prediction coefficients $\boldsymbol{\theta}_{t, T}^{*}$ by the local ones $\boldsymbol{\theta}_{t / T}$. Its proof is postponed to appendix B.1 for convenience.

Lemma 2.7. Let $d \in \mathbb{N}^{*}, \beta>0, R>0$ and $f_{-}>0$. Suppose that Assumption (M1) holds. Then, there exist two constants $C_{1}, T_{0}>0$ depending only on $d, \beta, R$ and $f_{-}$such that, for all $t \in \mathbb{Z}$ and $T \geq T_{0}$,

$$
\left\|\boldsymbol{\theta}_{t, T}^{*}-\boldsymbol{\theta}_{t / T}\right\| \leq C_{1} T^{-\min (1, \beta)} .
$$

An estimator $\widehat{\boldsymbol{\theta}}$ of $\boldsymbol{\theta}$ is studied in Dahlhaus and Giraitis (1998) for the model of Example 2.4. In the following we improve these results by deriving minimax rate properties of the estimator of Dahlhaus and Giraitis (1998) and extensions of it in a more general setting.

In the following, the problem that we are interested is to derive a minimax rate estimator $\widetilde{\boldsymbol{\theta}}$ at a given smoothness index $\beta>0$, which means that, for such a $\beta$, the estimation risk, say the quadratic risk $\mathbb{E}\left[\left\|\widetilde{\boldsymbol{\theta}}_{t, T}-\boldsymbol{\theta}_{t, T}^{*}\right\|^{2}\right]$, can be bounded uniformly over all processes satisfying ( $\mathrm{M}-1)$ (among with additional assumptions), and that the corresponding rate of convergence as $T \rightarrow \infty$ cannot be improved by any other estimator. The case $\beta \leq 2$ is solved in Moulines et al. (2005) for the subclass of TVAR models. 
2.3. Minimax estimation for adaptive prediction. Let $\widehat{X}_{d, t, T}^{*}$ denote the best linear predictor of order $d$ of $X_{t, T}$, which as a consequence of (2.10), reads

$$
\widehat{X}_{d, t, T}^{*}=\left(\boldsymbol{\theta}_{t, T}^{*}\right)^{\prime} \mathbf{X}_{t-1, T},
$$

We denote by $\widehat{X}_{t, T}^{*}$ the best predictor of $X_{t, T}$ given its past, that is, the conditional expectation

$$
\widehat{X}_{t, T}^{*}=\mathbb{E}\left[X_{t, T} \mid X_{s, T}, s \leq t-1\right] .
$$

As explained before, the goal of this paper is to derive estimators, say $\widetilde{\boldsymbol{\theta}}_{t, T} \in \mathbb{R}^{d}$, of $\boldsymbol{\theta}_{t / T}$, which is a local approximation of $\boldsymbol{\theta}_{t, T}^{*}$. In this section, we assume that $\widetilde{\boldsymbol{\theta}}_{t, T}$ is a function of the past $X_{s, T}, s \leq t-1$. Then $\widetilde{\boldsymbol{\theta}}_{t, T}^{\prime} \mathbf{X}_{t-1, T}$ is a legitimate predictor of $X_{t, T}$ and we have the following decomposition of the corresponding prediction quadratic risk

$$
\mathbb{E}\left[\left(X_{t, T}-\widetilde{\boldsymbol{\theta}}_{t, T}^{\prime} \mathbf{X}_{t-1, T}\right)^{2}\right]=\mathbb{E}\left[\left(X_{t, T}-\widehat{X}_{t, T}^{*}\right)^{2}\right]+\mathbb{E}\left[\left(\widetilde{\boldsymbol{\theta}}_{t, T}^{\prime} \mathbf{X}_{t-1, T}-\widehat{X}_{t, T}^{*}\right)^{2}\right] .
$$

The first term is the minimal prediction error that one would achieve with the conditional expectation (which requires the true distribution of the whole process). Furthermore, inserting $\widehat{X}_{d, t, T}^{*}=\left(\boldsymbol{\theta}_{t, T}^{*}\right)^{\prime} \mathbf{X}_{t-1, T}$ and using the Minkowskii and Cauchy-Schwartz inequalities, the square root of the second term can be bounded as

$$
\begin{aligned}
\left(\mathbb{E}\left[\left(\widetilde{\boldsymbol{\theta}}_{t, T}^{\prime} \mathbf{X}_{t-1, T}-\widehat{X}_{t, T}^{*}\right)^{2}\right]\right)^{1 / 2} \leq\left(\mathbb{E}\left[\left(\widehat{X}_{d, t, T}^{*}-\widehat{X}_{t, T}^{*}\right)^{2}\right]\right)^{1 / 2} & \\
& +\left(\mathbb{E}\left[\left\|\mathbf{X}_{t-1, T}\right\|^{4}\right]\right)^{1 / 4}\left(\mathbb{E}\left[\left\|\widetilde{\boldsymbol{\theta}}_{t, T}-\boldsymbol{\theta}_{t, T}^{*}\right\|^{4}\right]\right)^{1 / 4} .
\end{aligned}
$$

The first term in the upper bound is due to the approximation of the best predictor by the best linear predictor of order $d$ and can only be improved by increasing $d$. Note that, in the case of the $\operatorname{TVAR}(p)$ model with $p \leq d$, this error term vanishes. The quantity $\mathbb{E}\left[\left\|\mathbf{X}_{t-1, T}\right\|^{4}\right]$ is typically bounded by a constant independent of $(t, T)$ over the class of processes under consideration. Hence, for a given $d$, the control of the prediction risk boils down to the control of the estimation risk $\mathbb{E}\left[\left\|\widetilde{\boldsymbol{\theta}}_{t, T}-\boldsymbol{\theta}_{t, T}^{*}\right\|^{4}\right]$.

To do so, we can further decompose the loss as

$$
\left\|\widetilde{\boldsymbol{\theta}}_{t, T}-\boldsymbol{\theta}_{t, T}^{*}\right\| \leq\left\|\widetilde{\boldsymbol{\theta}}_{t, T}-\boldsymbol{\theta}_{t / T}\right\|+\left\|\boldsymbol{\theta}_{t / T}-\boldsymbol{\theta}_{t, T}^{*}\right\| .
$$

Note that the second term is a deterministic error basically accounting for the approximation precision of the non-stationary model by a stationary one, a bound of which is provided in Lemma 2.7 stated above.

As a result of the successive error developments above, our efforts in the following focus on controlling the estimation risk $\mathbb{E}\left[\left\|\widetilde{\boldsymbol{\theta}}_{t, T}-\boldsymbol{\theta}_{t / T}\right\|^{4}\right]$ uniformly over a class of weakly locally stationary processes with given smoothness index $\beta>0$.

2.4. Tapered Yule-Walker estimate. Following Dahlhaus and Giraitis (1998), a local empirical covariance function is defined as follows. It relies on a real data taper function $h$ and a bandwidth $M$ which may depend on $T$. 
Definition 2.8 (Local empirical covariance function). Consider a function $h$ : $[0,1] \rightarrow \mathbb{R}$ and $M \in 2 \mathbb{N}^{*}$. The empirical local covariance function $\widehat{\gamma}_{T, M}$ with taper $h$ is defined in $\mathbb{R} \times \mathbb{Z}$ as

$$
\widehat{\gamma}_{T, M}(u, \ell)=\frac{1}{H_{M}} \sum_{\substack{t_{1}, t_{2}=1 \\ t_{1}-t_{2}=\ell}}^{M} h\left(\frac{t_{1}}{M}\right) h\left(\frac{t_{2}}{M}\right) X_{\lfloor u T\rfloor+t_{1}-M / 2, T} X_{\lfloor u T\rfloor+t_{2}-M / 2, T},
$$

where $H_{M}=\sum_{k=1}^{M} h^{2}(k / M) \sim M \int_{0}^{1} h^{2}(x) \mathrm{d} x$ is the normalizing factor. If $H_{M}=0$, we set $\widehat{\gamma}_{T, M}(u, \ell)=0$, by convention.

For $h \equiv 1$ in Definition 2.8 we obtain the classical covariance estimate for a centred sample $\left\{X_{s},\lfloor u T\rfloor-M / 2 \leq s \leq\lfloor u T\rfloor+\ell+M / 2\right\}$. For any $d \geq 1$, based on the local empirical covariance function $\widehat{\gamma}_{T, M}$, the $d$-order local empirical YuleWalker prediction coefficients are then defined as

$$
\widehat{\boldsymbol{\theta}}_{t, T}(M)=\widehat{\Gamma}_{t, T, M}^{-1} \widehat{\gamma}_{t, T, M},
$$

where $\widehat{\gamma}_{t, T, M}=\left[\widehat{\gamma}_{T, M}(t / T, 1) \ldots \widehat{\gamma}_{T, M}(t / T, d)\right]^{\prime}, \widehat{\Gamma}_{t, T, M}$ is the matrix of empirical covariances $\widehat{\Gamma}_{t, T, M}=\left(\widehat{\gamma}_{T, M}(t / T, i-j) ; i, j=1, \ldots, d\right)$. The only way $\widehat{\Gamma}_{t, T, M}$ can be singular is when $\widehat{\gamma}_{T, M}(t / T, \ell)=0$ for all $\ell \in \mathbb{Z}$ (see Lemma A.5), in which case we just set $\widehat{\boldsymbol{\theta}}_{t, T}(M):=0$. Hence $\widehat{\boldsymbol{\theta}}_{t, T}(M)$ is always well defined and always satisfies the following (see again Lemma A.5 for the bound)

$$
\widehat{\Gamma}_{t, T, M} \widehat{\boldsymbol{\theta}}_{t, T}(M)=\widehat{\boldsymbol{\gamma}}_{t, T, M}, \quad \text { and } \quad\left\|\widehat{\boldsymbol{\theta}}_{t, T}(M)\right\| \leq 2^{d}-1 .
$$

Using this trick, we do not find it necessary to add additional assumptions on the model to guarantee that $\widehat{\Gamma}_{t, T, M}$ is non-singular a.s., as done for instance in Dahlhaus and Giraitis (1998), where $\mathbb{P}\left(X_{t, T}=0\right)=0$ for all $t \in \mathbb{Z}$ is assumed.

\section{Main results in the general framework}

3.1. Additional notation and assumptions. For convenience, we introduce the following notation. Let $p>0, q, r, s \in \mathbb{N}^{*}, \mu$ be a probability distribution on $\mathbb{R}, u: \mathbb{R} \rightarrow \mathbb{R}, a, b: \mathbb{R}^{r} \rightarrow \mathbb{R}, c \in \mathbb{R}^{q}$ and a collection of random matrices $\left\{U_{M} \in \mathbb{R}^{r \times s}, M \in \mathbb{N}^{*}\right\}$. We write

(i) $U_{M}=O_{L^{p}, \boldsymbol{c}}(u(M))$ if there exists $C_{p, \boldsymbol{c}}>0$, depending continuously and at most on $\left(p, \boldsymbol{c}^{\prime}\right)$, such that for all $M \in \mathbb{N}^{*}$

$$
\max _{1 \leq i \leq r, 1 \leq j \leq s}\left(\mathbb{E}\left[\left|U_{M i, j}\right|^{p}\right]\right)^{1 / p} \leq C_{p, c}|u(M)|,
$$

where $U_{M, i, j}$ is the $(i, j)$-th entry of the matrix $U_{M}$.

(ii) $U_{M}=O_{L} \bullet(\mu), c(u(M))$ if $U_{M}=O_{L^{p}, m_{p}, c}(u(M))$ for all $p \in[1, \infty)$, where $m_{p}$ is a constant only depending on the absolute moments of the distribution $\mu, \int|x|^{q} \mu(\mathrm{d} x), q \geq 1$.

(iii) $a(\boldsymbol{x})=O_{\boldsymbol{c}}(b(\boldsymbol{x}))$ if and only if there exists a constant $C_{\boldsymbol{c}}$ depending continuously and at most on the index $\boldsymbol{c}$, such that for all $\boldsymbol{x} \in \mathbb{R}^{r}$

$$
|a(\boldsymbol{x})| \leq C_{\boldsymbol{c}}|b(\boldsymbol{x})| .
$$

Concerning the function $h$ we have the following assumption. 
(H) The function $h:[0,1] \rightarrow \mathbb{R}$ is piecewise continuously differentiable, that is, for $0=u_{0}<u_{1}<\ldots<u_{N}=1, h$ is $\mathcal{C}^{1}$ on $\left(u_{i-1}, u_{i}\right], i=1, \ldots, N$. Moreover we assume $\int_{0}^{1} h^{2}=1,\|h\|_{\infty}=\sup _{u \in[0,1]}|h(u)|<\infty$ and $\left\|h^{\prime}\right\|_{\infty}=$ $\max _{1 \leq i \leq N} \sup _{u \in\left(u_{i-1}, u_{i}\right]}\left|h^{\prime}(u)\right|<\infty$.

Provided a piecewise continuously differentiable funtion $h($ as in $(\mathbf{H}))$ and a local spectral density function $f$ continuously differentiable on its first argument, we also consider the following assumption, which depends on a constant $C>0$ and on a probability distribution $\mu$ on $\mathbb{R}$.

(C) For all $\ell \in \mathbb{Z}$ and $h$ satisfying (H), we have, for all $t \in \mathbb{Z}$ and $T \geq T_{0}$,

$$
\widehat{\gamma}_{T, M}(u, \ell)-\mathbb{E}\left[\widehat{\gamma}_{T, M}(u, \ell)\right]=O_{L} \cdot(\mu), \ell,\|h\|_{\infty},\left\|h^{\prime}\right\|_{\infty}, C\left(M^{-1 / 2}\right) .
$$

Assumption (C) amounts to say that the tapered empirical covariance estimator $\widehat{\gamma}_{T, M}$ from a sample of length $M$ satisfies a standard deviation rate $M^{-1 / 2}$ in all $L^{q}$-norms. Locally stationary processes of Example 2.4 satisfy it under suitable assumptions (see Dahlhaus and Giraitis, 1998, Eq. (4.4) in Theorem 4.1). We conclude this section with a result that can be used for processes of Example 2.5.

Theorem 3.1. Let $\left(X_{t, T}\right)_{t \in \mathbb{Z}, T \geq T_{0}}$ be an array of random variables defined as in (2.6) where $\left(\xi_{t}\right)_{t \in \mathbb{Z}}$ are i.i.d. satisfying $\mathbb{E}\left[\left|\xi_{0}\right|^{q}\right]<\infty$ for all $q \geq 1$ and $\varphi_{t, T}^{0}$ satisfies (2.7) for some $\left(\psi_{k}\right)_{k \in \mathbb{N}} \in \ell_{+}^{1}(\mathbb{N}), K>0$ and $r \geq 1$. Assume moreover that there exist $k_{0} \in \mathbb{N},\left(\zeta_{k}\right)_{k \in \mathbb{N}} \in \ell_{+}^{1}(\mathbb{N})$ such that for all $t \in \mathbb{Z}, T \geq T_{0}$ and all $\boldsymbol{x}, \boldsymbol{x}^{\prime} \in \mathbb{R}^{\mathbb{N}}$ satisfying $x_{k}=x_{k}^{\prime}$ for $1 \leq k \leq k_{0}$,

$$
\left|\varphi_{t, T}^{0}(\boldsymbol{x})-\varphi_{t, T}^{0}\left(\boldsymbol{x}^{\prime}\right)\right| \leq K\left(\sum_{k \geq 0} \zeta_{k}\left|x_{k_{0}+k}-x_{k_{0}+k}^{\prime}\right|\right)\left(1+\sum_{k \geq 0} \psi_{k}\left(\left|x_{k}\right|+\left|x_{k}^{\prime}\right|\right)\right)^{r-1} .
$$

Suppose moreover that

$$
\sum_{k \geq 0} k \zeta_{k}<\infty
$$

Then there exists a constant $C$ only depending on $r, K, k_{0},\left(\psi_{k}\right)_{k \in \mathbb{N}},\left(\zeta_{k}\right)_{k \in \mathbb{N}}$ and the distribution of $\xi_{0}$ such that (C) holds.

The proof is postponed to Appendix C.1.

3.2. Bound of the estimation risk. Our first result on the estimation risk is a uniform approximation for the estimation error of $\widehat{\boldsymbol{\theta}}_{t, T}(M)$.

Theorem 3.2. Suppose that Assumption (M-1) holds with some $\beta>0, f_{-}>0$ and $R>0$, and let $h:[0,1] \rightarrow \mathbb{R}$ satisfying $(\mathbf{H})$. Let $k \in \mathbb{N}$ and $\alpha \in(0,1]$ be uniquely defined by the relation $\beta=k+\alpha$. Suppose that Assumption (C) holds for some constant $C>0$ and distribution $\mu$. Then, for any $d \geq 1$, the estimator $\widehat{\boldsymbol{\theta}}_{t, T}(M)$ defined by (2.16) satisfies

$$
\begin{aligned}
\widehat{\boldsymbol{\theta}}_{t, T}(M)-\boldsymbol{\theta}_{t / T}= & \sum_{\ell=1}^{k} \boldsymbol{a}_{h, f, \ell}\left(\frac{M}{T}\right)^{\ell} \\
& +O_{d, f_{-},\|h\|_{\infty},\left\|h^{\prime}\right\|_{\infty}, \beta, R}\left(\frac{1}{M}+\left(\frac{M}{T}\right)^{\beta}\right)+\boldsymbol{v}_{M},
\end{aligned}
$$


where $\boldsymbol{a}_{h, f, \ell} \in \mathbb{R}^{d}$ depends only on $h, f$ and $\ell$ and $\boldsymbol{v}_{M}=$

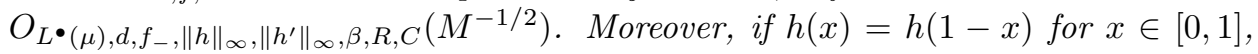
then $\boldsymbol{a}_{h, f, 1}=0$.

The proof is postponed to Appendix B.3.

Remark 3.3. In (3.4), the choice of the taper may influence the rate of convergence through the constant $\boldsymbol{a}_{h, f, 1}$, which vanishes if the taper is symmetric, that is, if $h(x)=h(1-x)$ for $x \in[0,1]$. Other constants depend on the choice of the taper but one cannot choose tapers that ensure a further systematic improvement of the rate. The reason is given by the definition of the constant $c_{h, 2}$ appearing in the proof of Theorem B.2, which implies $c_{h, 2}>0$ for all tapers $h$. Consequently, for any taper $h$, one have that $\boldsymbol{a}_{h, f, 2} \neq 0$, except perhaps for some particular local density functions $f$. Hence, as far as rates of convergence are concerned, the only important property of the taper is that of being symmetric.

Theorem 3.2 suggests to combine several $\widehat{\boldsymbol{\theta}}_{t, T}(M)$ to obtain a more accurate estimation by canceling out the first $k$ bias terms in (3.4). This technique was already used for eliminate one term of bias in Moulines et al. (2005, Theorem 8). It is inspired by the Romberg's method in numerical analysis (see Baranger and Brezinski, 1991). Let $\boldsymbol{\omega}=\left[\omega_{0} \ldots \omega_{k}\right]^{\prime} \in \mathbb{R}^{k+1}$, be the solution of the equation

$$
A \omega=e_{1},
$$

where $\boldsymbol{e}_{1}=\left[\begin{array}{llll}1 & 0 & \ldots & 0\end{array}\right]^{\prime}$ is the $\mathbb{R}^{k+1}$ - vector having a 1 in the first position and zero everywhere else and $A$ is a $(k+1) \times(k+1)$ matrix with entries $A_{i, j}=2^{i j}$ for $0 \leq i, j \leq k$.

Theorem 3.4. Under the same assumptions as Theorem 3.2, the estimator

$$
\widetilde{\boldsymbol{\theta}}_{t, T}(M)=\sum_{j=0}^{k} \omega_{j} \widehat{\boldsymbol{\theta}}_{t, T}\left(2^{j} M\right),
$$

with $\boldsymbol{\omega}$ defined by (3.5), satisfies

$$
\begin{aligned}
& \widetilde{\boldsymbol{\theta}}_{t, T}(M)-\boldsymbol{\theta}_{t / T}= O_{d, f_{-},\|h\|_{\infty},\left\|h^{\prime}\right\|_{\infty}, \beta, R}\left(\frac{1}{M}+\left(\frac{M}{T}\right)^{\beta}\right) \\
&+O_{L} \bullet(\mu), d, f_{-},\|h\|_{\infty},\left\|h^{\prime}\right\|_{\infty}, \beta, R, C \\
&\left(M^{-1 / 2}\right) .
\end{aligned}
$$

The proof is postponed to Appendix B.4.

Remark 3.5. If $h(x)=h(1-x)$ for $x \in[0,1]$ then the first order term of $(3.4)$ is zero; in this case we can remove the term $j=k$ in (3.6) and define $\boldsymbol{\omega}=$ $\left[\begin{array}{lll}\omega_{0} & \ldots & \omega_{k-1}\end{array}\right]^{\prime} \in \mathbb{R}^{k}$ by (3.5) with the second row and last column of $A$ removed and $\boldsymbol{e}_{1}=\left[\begin{array}{llll}1 & 0 & \ldots & 0\end{array}\right]^{\prime} \in \mathbb{R}^{k}$.

It is straightforward to check that the optimal bandwidth for minimizing the order of the right term of Equation (3.7) is $M \propto T^{2 \beta /(2 \beta+1)}$, yielding the next result.

Corollary 3.6. Let $\beta, R, f_{-}>0$ and $h:[0,1] \rightarrow \mathbb{R}$. Let $k \in \mathbb{N}$ and $\omega \in(0,1]$ be uniquely defined such that $\beta=k+\omega$ and set $M:=2\left\lfloor T^{2 \beta /(2 \beta+1)}\right\rfloor$ in the following. Suppose that Assumptions (M-1), (H) and (C) hold. Let $\widetilde{\boldsymbol{\theta}}_{t, T}(M)$ be obtained as in Theorem 3.4. Then, for any $q>0$ there exist a constant $C_{0}$ only depending on 
$h, q, d, f_{-}, R, \mu$ and $\beta$, and $a T_{0}>0$ only depending on $d, R, \beta, f_{-}$and $C$ such that, for all $T \geq T_{0}$ and all $t \in \mathbb{Z}$,

$$
\left(\mathbb{E}\left[\left\|\widetilde{\boldsymbol{\theta}}_{t, T}(M)-\boldsymbol{\theta}_{t / T}\right\|^{q}\right]\right)^{1 / q} \leq C_{0} T^{-\beta /(2 \beta+1)} .
$$

It is interesting to note that in the decomposition (2.15), the bound of the error term $\left\|\widetilde{\boldsymbol{\theta}}_{t, T}(M)-\boldsymbol{\theta}_{t / T}\right\|$ in (3.8) always has a slower decaying rate that that of the bound of the (deterministic) error term $\left\|\boldsymbol{\theta}_{t / T}-\boldsymbol{\theta}_{t, T}^{*}\right\|$ in Lemma 2.7.

\section{Application to TVAR processes}

TVAR processes (see Example 2.6) are a handful model to illustrate our results. Under suitable assumptions, they have the specific property that, when $d=p$, the linear predictor coefficients in $\boldsymbol{\theta}_{t, T}^{*} \in \mathbb{R}^{d}$ as defined by Equation (2.10) coincide with the time-varying autoregressive coefficients given by $\boldsymbol{\theta}(t / T)$ of the $\operatorname{TVAR}(p)$ equation (2.9) and also with the local solution $\boldsymbol{\theta}_{t / T}$ of the Yule-Walker equations defined by (2.12), see (4.4) below.

In the following, we introduce some smoothness assumptions on the time-varying parameters, similar to (and actually yielding) the one required on the local spectral density in 2.3. Additional stability conditions are also required, based on the

following definitions. For $\boldsymbol{\theta}: \mathbb{R} \rightarrow \mathbb{R}^{p}, u \mapsto\left[\begin{array}{lll}\theta_{1}(u) & \ldots & \theta_{p}(u)\end{array}\right]^{\prime}$ we define the time varying autoregressive polynomial by

$$
\boldsymbol{\theta}(z ; u):=1-\sum_{j=1}^{p} \theta_{j}(u) z^{j} .
$$

Let us define, for any $p \in \mathbb{N}^{*}$ and $\delta>0$,

$$
\begin{aligned}
s_{(p)}(\delta) & :=\left\{\boldsymbol{\theta}=\left[\begin{array}{lll}
\theta_{1} & \ldots & \theta_{p}
\end{array}\right]^{\prime} \in \mathbb{R}^{p} \text { s.t. } 1-\sum_{j=1}^{p} \theta_{j} z^{j} \neq 0, \forall|z|<\delta^{-1}\right\}, \\
s_{p}(\delta) & :=\left\{\boldsymbol{\theta}: \mathbb{R} \rightarrow s_{(p)}(\delta)\right\} \\
& =\left\{\boldsymbol{\theta}: \mathbb{R} \rightarrow \mathbb{R}^{p} \text { s.t. } \boldsymbol{\theta}(z ; u) \neq 0, \forall|z|<\delta^{-1}, u \in \mathbb{R}\right\} .
\end{aligned}
$$

Define, for $\beta>0, R>0, \delta \in(0,1), \rho \in[0,1]$ and $\sigma_{+}>0$, the class of parameters

$$
\begin{gathered}
\mathcal{C}\left(\beta, R, \delta, \rho, \sigma_{+}\right)=\left\{(\boldsymbol{\theta}, \sigma): \mathbb{R} \rightarrow \mathbb{R}^{p} \times\left[\rho \sigma_{+}, \sigma_{+}\right] \text {s.t. } \boldsymbol{\theta} \in \Lambda_{p}(\beta, R) \cap s_{p}(\delta),\right. \\
\left.\sigma \in \Lambda_{1}(\beta, R)\right\} .
\end{gathered}
$$

The first result to provide sufficient conditions on the TVAR coefficients for the existence of a stable solution of the TVAR equations goes back to Künsch (1995). Here we use Giraud et al. (2015, Proposition 1), which guarantees the following: given a centered i.i.d. sequence $\left(\xi_{t}\right)_{t \in \mathbb{Z}}$ with unit variance and given the constants $\delta \in(0,1), \rho \in[0,1], \sigma_{+}>0, \beta>0$ and $R>0$, there exists a large enough $T_{0}$ only depending on $\delta, \beta$ and $R$ such that, for all $(\boldsymbol{\theta}, \sigma) \in \mathcal{C}\left(\beta, R, \delta, \rho, \sigma_{+}\right)$, there exists a unique process $\left(X_{t, T}\right)_{t \in \mathbb{Z}, T \geq T_{0}}$ satisfying (2.9) for all $t \in \mathbb{Z}$ and $T \geq T_{0}$ and such that, for all $T \geq T_{0}, X_{t, T_{0}}$ is bounded in probability as $t \rightarrow-\infty$. We use this result as our definition of the TVAR process with time varying AR coefficients $\theta_{1}, \ldots, \theta_{p}$, time varying standard deviation $\sigma$, and innovations $\left(\xi_{t}\right)_{t \in \mathbb{Z}}$. For later reference, we summarize this in the following assumption. 
(M-2) Let $\left(\xi_{t}\right)_{t \in \mathbb{Z}}$ be an i.id. sequence with zero mean and unit variance. Assume that $(\boldsymbol{\theta}, \sigma) \in \mathcal{C}\left(\beta, R_{0}, \delta, \rho, \sigma_{+}\right)$with $\delta \in(0,1), \beta>0, R_{0}>0$ and $\rho \in[0,1]$. The array $\left(X_{t, T}\right)_{t \in \mathbb{Z}, T \geq T_{0}}$ is a TVAR process as previously defined with time varying AR coefficients $\theta_{1}, \ldots, \theta_{p}$, time varying standard deviation $\sigma$, and innovations $\left(\xi_{t}\right)_{t \in \mathbb{Z}}$

In this assumption the constant $T_{0}$ is set to have the existence and uniqueness of the stable solution of the TVAR equation for all $T \geq T_{0}$. It may change hereafter from line to line to guarantee additional properties of the solution but always in a way where it depends at most on the constants $\beta, R_{0}, \delta, \rho$ and $\sigma_{+}$.

The following assumption can be used to control the moments of any order of the TVAR process.

(I) For all $q>0$ the innovations $\left(\xi_{t}\right)_{t \in \mathbb{Z}}$ satisfy $\mathbb{E}\left[\left|\xi_{0}\right|^{q}\right]<\infty$.

Time varying autoregressive processes are well known to be locally stationary under certain conditions on their parameters and moments, see Dahlhaus (1996b, Theorem 2.3). Adapting these results to our context, we have the following.

Theorem 4.1. Assumption (M-2) implies the two following assertions.

(i) There exist constants $R$ and $T_{0}$ only depending on $p, \delta, \sigma_{+}, \beta$ and $R_{0}$ such that $\left(X_{t, T}\right)_{t \in \mathbb{Z}, T \geq T_{0}}$ is $(\beta, R)$-weakly locally stationary in the sense of Definition 2.3 with local spectral density defined by

$$
f(u, \lambda)=\frac{\sigma^{2}(u)}{2 \pi}\left|\boldsymbol{\theta}\left(\mathrm{e}^{-\mathrm{i} \lambda} ; u\right)\right|^{-2} .
$$

Moreover, we have, for all $T \geq T_{0}$ and $t \in \mathbb{Z}$,

$$
\boldsymbol{\theta}(t / T)=\boldsymbol{\theta}_{t, T}^{*}=\boldsymbol{\theta}_{t / T},
$$

where $\boldsymbol{\theta}_{t, T}^{*}$ and $\boldsymbol{\theta}_{t / T}$ are the optimal and local prediction coefficients respectively defined by (2.10) and (2.12) in the case $d=p$.

(ii) If $\rho \in(0,1]$, then Assumption ( $\mathrm{M}-1)$ holds with the same $\beta$ and some constants $R, T_{0}$ and $f_{-}>0$ only depending on $p, \delta, \rho, \sigma_{+}, \beta$ and $R_{0}$.

(iii) If $\mathbb{P}\left(\xi_{0}=x\right)=0$ for all $x \in \mathbb{R}$, then $\mathbb{P}\left(X_{t, T}=0\right)=0$ for all $t \in \mathbb{Z}$ and $T \geq T_{0}$.

(iv) If (I) holds, then $\left(X_{t, T}\right)_{t \in \mathbb{Z}, T \geq T_{0}}$ satisfies Assumption (C) with $C$ only depending on $R_{0}, \beta, \delta, \sigma_{+}$and with $\mu$ defined as the distribution of $\xi_{0}$.

The proof is postponed to Appendix C.2. Theorem 4.1 basically shows that the results of Section 3 apply to TVAR processes, as defined by ( $\mathrm{M}-2)$ provided that $\rho>0$ and $(\mathrm{I})$ is assumed on the innovations. We specifically state the following result which provides a useful complement to Moulines et al. (2005, Corollary 9) where the same minimax rate is exhibited for a different estimator but only for smoothness index $\beta \leq 2$.

Corollary 4.2. Let $\delta \in(0,1), \beta>0, R>0$ and $\rho \in(0,1]$. Suppose that Assumptions (M-2) and (I) hold. Let $M=2\left\lfloor T^{2 \beta /(2 \beta+1)}\right\rfloor$ and $\widetilde{\boldsymbol{\theta}}_{t, T}(M)$ be the estimator defined by (2.16) and (3.6) with $p$, the order of the TVAR process equal to $d$, the order of the prediction vector. Then, for any $q \in \mathbb{N}$ there exists a constant $C$ only depending on $q, h, p, \delta, \rho, \sigma_{+}, \beta, R_{0}$ and the moments of the distribution of $\xi_{0}$ such that, for all $T \geq T_{0}$ and $t \in \mathbb{Z}$, we have

$$
\left(\mathbb{E}\left[\left\|\widetilde{\boldsymbol{\theta}}_{t, T}(M)-\boldsymbol{\theta}\left(\frac{t}{T}\right)\right\|^{q}\right]\right)^{1 / q} \leq C T^{-\beta /(2 \beta+1)} .
$$


Proof: By Theorem 4.1, we can apply Corollary 3.6. Recall that in the case of the TVAR with order $p$ equal to the prediction length, we have $\boldsymbol{\theta}(t / T)=\boldsymbol{\theta}_{t / T}=$ $\boldsymbol{\theta}_{t, T}^{*}$.

The estimator $\widetilde{\boldsymbol{\theta}}$ proposed in Corollary 4.2 achieves the $\beta$-minimax-rate for TVAR processes according to the lower Moulines et al. (2005, Theorem 4). Hence, it is also $\beta$-minimax-rate in the class of weakly locally stationary processes satisfying Assumption (M-1). Giraud et al. (2015, Section A.1) explains how to construct minimax-rate predictors from minimax-rate estimators of $\boldsymbol{\theta}$. Applying their approach, Corollary 4.2 also provides a crucial ingredient in building $\beta$-minimax-rate predictors for any $\beta>0$.

\section{Numerical work}

We test both methods on data simulated according to a TVAR process with $p=3$. The parameter function $u \mapsto \boldsymbol{\theta}(u)$ within $s_{p}(\delta)$ for some $\delta \in(0,1)$ is chosen randomly as follows. First we pick randomly some smoothly time varying partial autocorrelation functions up to the order $p$ that are bounded between -1 and $1, \check{\theta}_{k, k}(u) \propto \sum_{j=1}^{F-1} a_{j, k} j^{2} \cos (j u)$, where $a_{j, k}$ are random numbers in $[-1,1]$. Here $\check{\theta}_{k, k}(u)$ is defined up to a multiplicative constant; dividing, for example, by $F(F-1)(2 F-1) / 6$ guarantees its values to remain within $(-1,1)$. Then, for any required $t, T$, we use Algorithm 1 with input $\check{\theta}_{k, k}(t / T)$ and assign the output to $\boldsymbol{\theta}(t / T)$. Based on the classical Levinson-Durbin recurrence (see for example Brockwell and Davis (2006, Proposition 5.2.1)), the $\check{\boldsymbol{\theta}}$ in Algorithm 1 is in $s_{(p)}(1)$ as defined in (4.1), and it follows that the output $\boldsymbol{\theta} \in s_{(p)}(\delta)$. The randomly obtained three components of our $\boldsymbol{\theta}(t)$ are displayed in Figure 5.1, for $t \in[0,1]$.

For each $T \in\left\{2^{2 j}, j=5, \ldots, 15\right\}$ we generate 100 realizations of a TVAR process from innovation sequences $\left(\xi_{t}\right)_{t \in \mathbb{Z}}$ of i.i.d. centred Gaussian random variables with unit variance by sampling the previous $\boldsymbol{\theta}$ at a rate $T^{-1}$, and taking $\sigma \equiv 1$.

Then we compare $\widehat{\boldsymbol{\theta}}$ and $\widetilde{\boldsymbol{\theta}}$ for estimating $\boldsymbol{\theta}(1 / 2)$ using $h \equiv 1$ and different values of $M$. Recall that $\boldsymbol{\theta}(1 / 2)=\boldsymbol{\theta}_{T / 2, T}^{*}$. Figure 5.2 shows the boxplots corresponding to this evaluation for two different $T$ s. In Figure 5.2 we observe that for $T=2^{20}$ the estimation error of $\widehat{\boldsymbol{\theta}}$ is minimized in $M=2^{15}$ while that of $\widetilde{\boldsymbol{\theta}}$ is minimized in $M=2^{17}$. The estimator $\widetilde{\boldsymbol{\theta}}$ beats $\widehat{\boldsymbol{\theta}}$ for the two biggest values of $M$. In the case $T=2^{30}$, the error of $\widehat{\boldsymbol{\theta}}$ reaches its minimum in $M=2^{23}$ and that of $\tilde{\boldsymbol{\theta}}$ in $M=2^{26}$. The estimator $\widetilde{\boldsymbol{\theta}}$ beats $\widehat{\boldsymbol{\theta}}$ for the four biggest values of $M$. These experiences illustrate the theoretical result established in Theorem 3.2 and Corollary 4.2 that after optimizing in $M, \widetilde{\boldsymbol{\theta}}$ outperforms $\widehat{\boldsymbol{\theta}}$ for $T$ large enough.

To corroborate these conclusions over a wider range of $T$ 's, we refer to Figure 5.3. The plot on the left-hand side shows the oracle errors $\min _{M}\left\|\widehat{\boldsymbol{\theta}}_{T / 2, T}(M)-\boldsymbol{\theta}(1 / 2)\right\|$ and $\min _{M}\left\|\widetilde{\boldsymbol{\theta}}_{T / 2, T}(M)-\boldsymbol{\theta}(1 / 2)\right\|$ for all $T \in\left\{2^{2 j}, 5 \leq j \leq 15\right\}$. The slope corresponding to $\boldsymbol{\theta}$ (in blue) is steeper than the one corresponding to $\widehat{\boldsymbol{\theta}}$ (in red), meaning that, in average, $\widetilde{\boldsymbol{\theta}}$ outperforms $\widehat{\boldsymbol{\theta}}$ by an increasing order of magnitude as $T$ increases. The boxplots on the right-hand side of Figure 5.3 represent the ratios $\min _{M}\left\|\widetilde{\boldsymbol{\theta}}_{T / 2, T}(M)-\boldsymbol{\theta}(1 / 2)\right\| / \min _{M}\left\|\widehat{\boldsymbol{\theta}}_{T / 2, T}(M)-\boldsymbol{\theta}(1 / 2)\right\|$ computed for each $T$ and realization of the TVAR process. Observe that for $2^{14} \leq T \leq 2^{18}$ the estimator $\widetilde{\boldsymbol{\theta}}$ beats $\widehat{\boldsymbol{\theta}}$ in at least half of the cases. For $T \geq 2^{20}$, it happens in at least 
$75 \%$ of the cases. We conclude that the estimator with reduced bias is of interest when the length of the data set becomes very large.
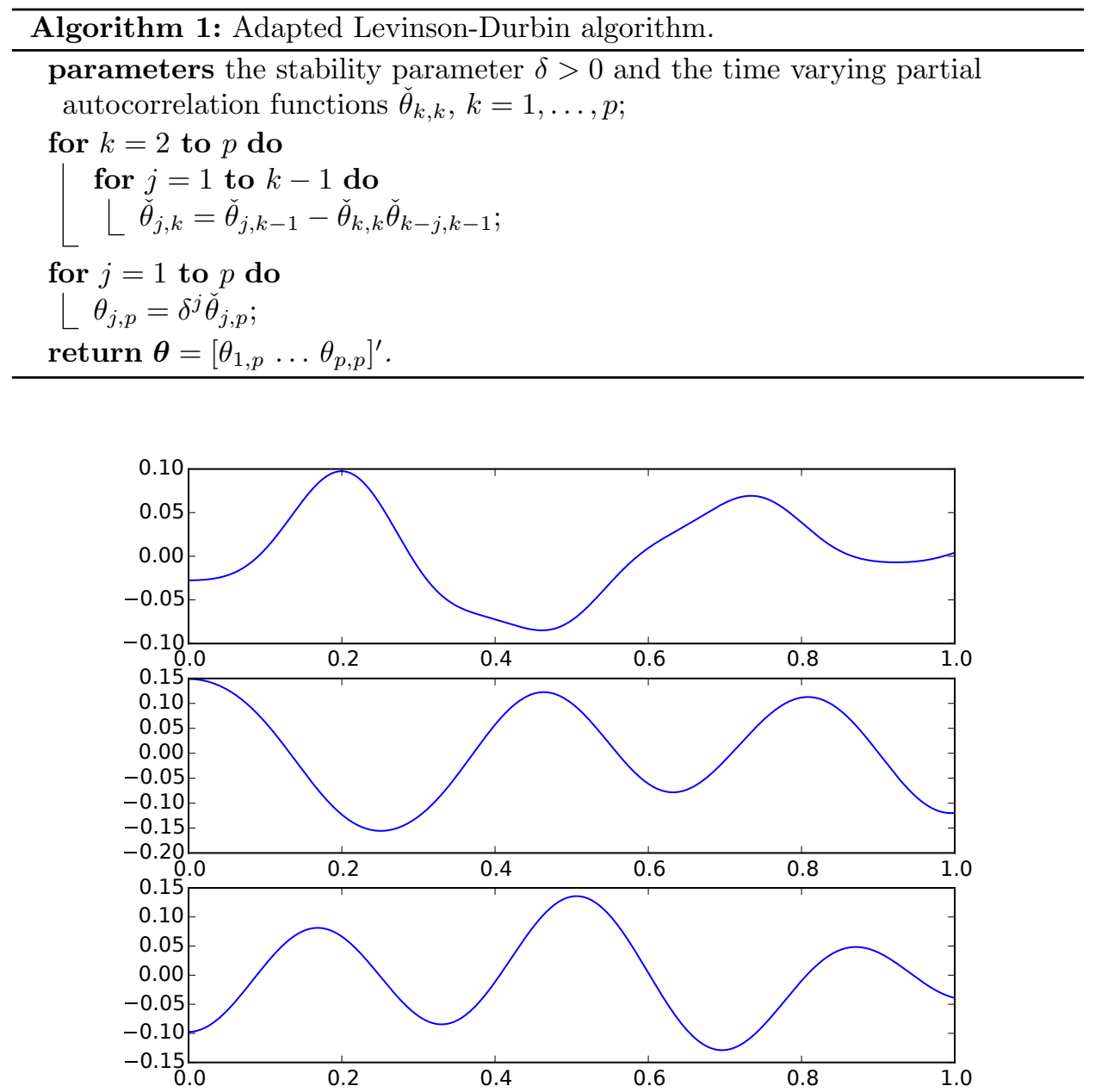

Figure 5.1. Plots of $\theta_{1}(t)$ (top), $\theta_{2}(t)$ (middle) and $\theta_{3}(t)$ (bottom) on the interval $t \in[0,1]$.

\section{Acknowledgments}

We thank Tobias Kley for his thoughtful reading of the paper and appropriate remarks (concerning specially Lemma 2.7) and also for bringing forward to our attention the related work Kley et al. (2016).

\section{Appendix A. Useful lemmas}

We gather here some useful lemmas that are (simple extensions of) standard results for Yule-Walker estimation of the linear prediction coefficients. Most of them are similar to those used in Dahlhaus and Giraitis (1998). Short proofs are 

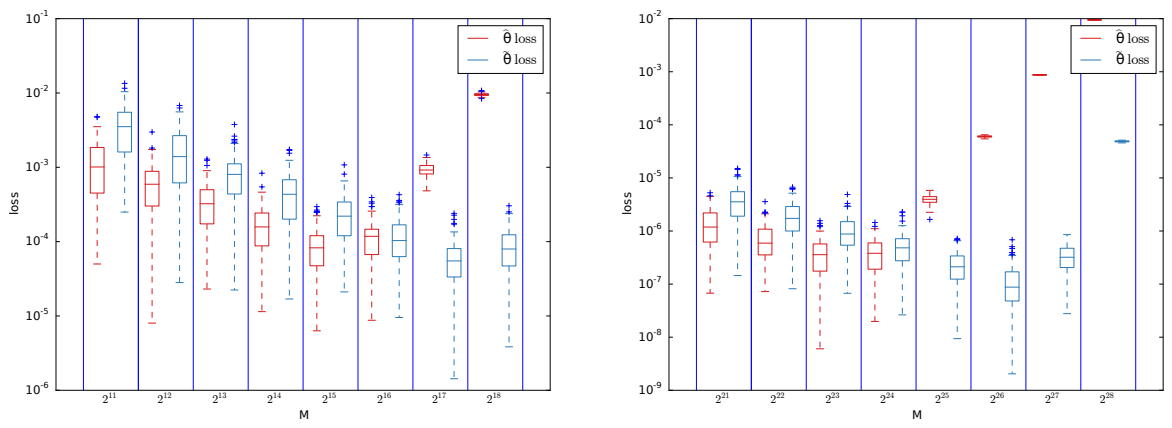

Figure 5.2. Box plots of the quadratic losses for estimating $\boldsymbol{\theta}(1 / 2)$ using $\widehat{\boldsymbol{\theta}}_{T / 2, T}(M)$ (red boxes) and $\widetilde{\boldsymbol{\theta}}_{T / 2, T}(M)$ (blue boxes) for various bandwidths $M$, when $T=2^{20}$ (left) and $T=2^{30}$ (right).
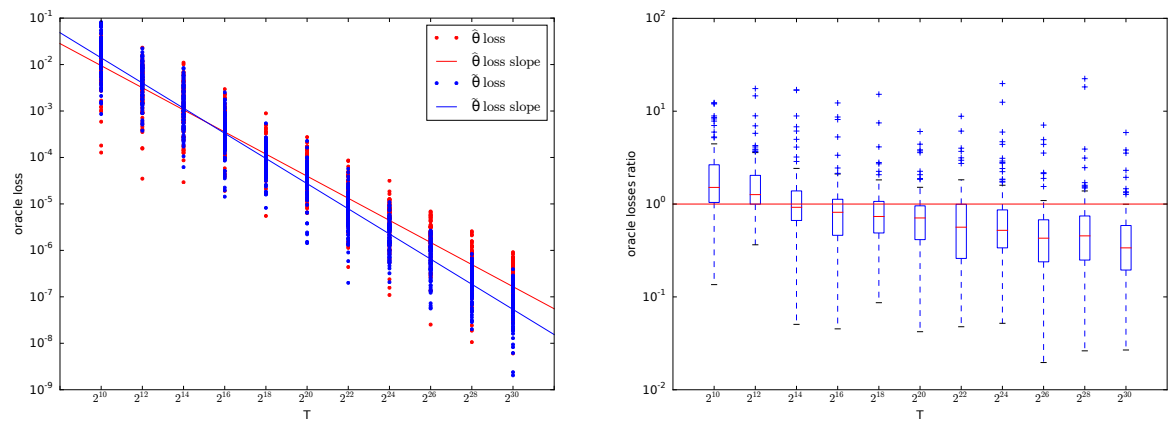

Figure 5.3. Oracle losses (using the best choice for the bandwidth $M)$ for estimating $\boldsymbol{\theta}(1 / 2)$ using $\widehat{\boldsymbol{\theta}}_{T / 2, T}(M)$ (red points) and $\widetilde{\boldsymbol{\theta}}_{T / 2, T}(M)$ (blue points) for various values of $T$. The left-hand side plot displays the losses over all the Monte Carlo simulations and the two resulting log-log regression lines. The right-hand side plot displays boxplots of the corresponding losses ratio.

provided for the sake of completeness. Different bounds can be found in Kley et al. (2016), in order to better control the case $d \rightarrow \infty$.

Lemma A.1. Let $d$ be a positive integer. Consider the $d \times d$ matrices $\Gamma$ and $\widehat{\Gamma}$ and vectors $\boldsymbol{\gamma}, \widehat{\boldsymbol{\gamma}}, \boldsymbol{\theta}, \widehat{\boldsymbol{\theta}} \in \mathbb{R}^{d}$ satisfying the relations

$$
\begin{aligned}
\Gamma \boldsymbol{\theta} & =\gamma, \\
\widehat{\Gamma} \widehat{\boldsymbol{\theta}} & =\widehat{\gamma} .
\end{aligned}
$$

Then, for any $k \in \mathbb{N}$ we have, if $\Gamma$ is non-singular,

$$
\begin{array}{r}
\widehat{\boldsymbol{\theta}}-\boldsymbol{\theta}=\left(\Gamma^{-1}+\sum_{\ell=1}^{k}\left(\Gamma^{-1}(\Gamma-\widehat{\Gamma})\right)^{\ell}\right)(\widehat{\gamma}-\gamma)+\sum_{\ell=1}^{k+1}\left(\Gamma^{-1}(\Gamma-\widehat{\Gamma})\right)^{\ell} \boldsymbol{\theta} \\
+\left(\Gamma^{-1}(\Gamma-\widehat{\Gamma})\right)^{k+1}(\widehat{\boldsymbol{\theta}}-\boldsymbol{\theta}) .
\end{array}
$$


Proof: From Equations (A.1) and (A.2) we get

$$
\widehat{\boldsymbol{\theta}}-\boldsymbol{\theta}=\Gamma^{-1}[(\Gamma-\widehat{\Gamma}) \widehat{\boldsymbol{\theta}}+\widehat{\gamma}-\gamma]
$$

The result follows by applying a recursion on $k=0,1, \ldots$.

Lemma A.2. Let $\gamma$ be the autocovariance function associated with a spectral density function $f, \gamma(s)=\int_{0}^{2 \pi} \mathrm{e}^{\mathrm{i} s \lambda} f(\lambda) \mathrm{d} \lambda$, for all $s \in \mathbb{Z}$, and denote by $\Gamma_{d}$ the corresponding covariance matrix of size $d \times d$,

$$
\Gamma_{d}=\left[\begin{array}{ccc}
\gamma(0) & \ldots & \gamma(d-1) \\
\vdots & \ddots & \vdots \\
\gamma(d-1) & \ldots & \gamma(0)
\end{array}\right]
$$

Then the following assertions hold for any $d \in \mathbb{N}^{*}$.

(i) If $\int_{0}^{2 \pi} f>0$ then $\Gamma_{d}$ is positive definite.

(ii) If $f$ is valued in $\left[f_{-}, f_{+}\right]$with $f_{-} \leq f_{+}$, then all the eigenvalues of $\Gamma_{d}$ belong to $\left[2 \pi f_{-}, 2 \pi f_{+}\right]$.

Proof: These well known facts (see e.g. Brockwell and Davis, 2006, Proposition 4.5.3) follow from the identity $\boldsymbol{a}^{\prime} \Gamma_{d} \boldsymbol{a}=\int_{-\pi}^{\pi}\left|\sum_{j=1}^{d} a_{j} \mathrm{e}^{\mathrm{i} j \lambda}\right|^{2} f(\lambda) \mathrm{d} \lambda$, for all $\boldsymbol{a}=\left[\begin{array}{lll}a_{1} & \ldots & a_{d}\end{array}\right]^{\prime} \in \mathbb{R}^{d}$

The next lemmas allow us to control the norms of $\widehat{\boldsymbol{\theta}}_{t, T}$ and $\boldsymbol{\theta}_{t / T}$.

Lemma A.3. Let $p$ be a positive integer and $\delta>0$. The set $s_{(p)}(\delta)$ defined in (4.1) is a closed subset of the ball $\left\{\boldsymbol{\theta} \in \mathbb{R}^{p}\right.$ s.t. $\left.\|\boldsymbol{\theta}\| \leq(1+\delta)^{p}-1\right\}$.

Proof: Hurwitz's theorem (see Conway, 1973, Theorem 2.5 or Gamelin, 2001, Section 3, Chapter VIII) implies that $s_{(p)}(\delta)$ is a closed subset of $\mathbb{R}^{p}$. It is also bounded (see Moulines et al., 2005, Lemma 1). Hereafter we provide a slightly different bound using Euclidean norm instead of the supnorm.

Take now $\boldsymbol{\theta} \in s_{(p)}(\delta)$. Let $z_{1}, \ldots, z_{p}$ denote the complex roots of the polynomial $\boldsymbol{\theta}(z):=1-\sum_{j=1}^{p} \theta_{j} z^{j}$. They satisfy $\left|z_{j}\right| \geq \delta^{-1}$ for any $j$. The following holds

$$
1+\|\boldsymbol{\theta}\|^{2}=\frac{1}{2 \pi} \int_{-\pi}^{\pi}\left|1-\sum_{j=1}^{p} \theta_{j} \mathrm{e}^{\mathrm{i} j \lambda}\right|^{2} \mathrm{~d} \lambda=\frac{1}{2 \pi} \int_{-\pi}^{\pi}\left|\boldsymbol{\theta}\left(\mathrm{e}^{\mathrm{i} \lambda}\right)\right|^{2} \mathrm{~d} \lambda .
$$

On the other hand we have $\boldsymbol{\theta}(z)=\prod_{j=1}^{p}\left(1-z z_{j}^{-1}\right)$, so that for $|z|=1$, since $\left|z_{j}\right|^{-1} \leq \delta$, we get $|\boldsymbol{\theta}(z)| \leq(1+\delta)^{p}$. Putting this into (A.5) the proof is completed.

The next lemma is similar in flavor to the statistical result of Whittle (1963, Section 3). It is also a classical property of orthogonal polynomials (see Grenander and Szegó, 1984, Section 2.4). We provide an elementary proof.

Lemma A.4. Let $\gamma$ be an autocovariance function. Let $d \geq 1$ such that the covariance matrix $\Gamma_{d}$ defined by Equation (A.4) is positive-definite. Let $\boldsymbol{\theta}$ denote the solution of the d-order Yule-Walker equation, $\boldsymbol{\theta}=\left[\theta_{1} \ldots \theta_{d}\right]^{\prime}=\Gamma_{d}^{-1} \gamma_{d}$ with $\gamma_{d}=[\gamma(1) \ldots \gamma(d)]^{\prime}$. Then we have $\boldsymbol{\theta} \in s_{(d)}(1)$ and $\|\boldsymbol{\theta}\| \leq 2^{d}-1$. 
Proof: We only need to prove $\boldsymbol{\theta} \in s_{(d)}(1)$ since $\|\boldsymbol{\theta}\| \leq 2^{d}-1$ is then implied by Lemma A.3 with $p=d$ and $\delta=1$.

For $j=1, \ldots, d$, let $\boldsymbol{e}_{j}=\left[\begin{array}{llllll}0 & \ldots & 1 & \ldots & 0\end{array}\right]^{\prime}$ be the $\mathbb{R}^{d}$ - vector having a 1 in the $j$-th position and zero everywhere else. Consider also the companion matrix $A=$ $\left[\begin{array}{llll}\boldsymbol{\theta} & \boldsymbol{e}_{1} & \ldots & \boldsymbol{e}_{d-1}\end{array}\right]^{\prime}$. Since the roots of $\boldsymbol{\theta}(z)$ are the inverses of the eigenvalues of $A$, or $A^{\prime}$, we only need to prove that the eigenvalues of $A^{\prime}$ are inside the closed unit disk. Observe that

$$
\Gamma_{d}-A \Gamma_{d} A^{\prime}=\Gamma_{d}-\left[\begin{array}{ccccc}
\boldsymbol{\theta}^{\prime} \Gamma_{d} \boldsymbol{\theta} & \boldsymbol{\theta}^{\prime} \Gamma_{d} \boldsymbol{e}_{1} & \ldots & \ldots & \boldsymbol{\theta}^{\prime} \Gamma_{d} \boldsymbol{e}_{d-1} \\
\boldsymbol{e}_{1}^{\prime} \Gamma_{d} \boldsymbol{\theta} & \boldsymbol{e}_{1}^{\prime} \Gamma_{d} \boldsymbol{e}_{1} & \ldots & \ldots & \boldsymbol{e}_{1}^{\prime} \Gamma_{d} \boldsymbol{e}_{d-1} \\
\boldsymbol{e}_{2}^{\prime} \Gamma_{d} \boldsymbol{\theta} & \boldsymbol{e}_{2}^{\prime} \Gamma_{d} \boldsymbol{e}_{1} & \ldots & \ldots & \boldsymbol{e}_{2}^{\prime} \Gamma_{d} \boldsymbol{e}_{d-1} \\
\vdots & \vdots & \ddots & \ddots & \vdots \\
\boldsymbol{e}_{d-1}^{\prime} \Gamma_{d} \boldsymbol{\theta} & \boldsymbol{e}_{d-1}^{\prime} \Gamma_{d} \boldsymbol{e}_{1} & \ldots & \ldots & \boldsymbol{e}_{d-1}^{\prime} \Gamma_{d} \boldsymbol{e}_{d-1}
\end{array}\right]
$$

Because $\Gamma_{d}$ is a Toeplitz matrix, its $(i, j)$-th entries, and those of $A \Gamma_{d} A^{\prime}$ are equal for $i, j \geq 2$. The definition of $\boldsymbol{\theta}$ implies also the equality of the $(i, j)$-th entries of both matrices when $i=1, j \geq 2$ and $i \geq 2, j=1$. Hence $\Gamma_{d}-A \Gamma_{d} A^{\prime}$ is a $d \times d$ symmetric matrix with zero entries except at the top-left where it takes value $\gamma(0)-\boldsymbol{\theta}^{\prime} \gamma_{d}$. This value is non-negative since it is the variance of the prediction error or order $d$. Hence we conclude that for $\boldsymbol{v} \in \mathbb{C}^{d}, \boldsymbol{v}^{\prime}\left(\Gamma_{d}-A \Gamma_{d} A^{\prime}\right) \boldsymbol{v} \geq 0$. Consider now $\lambda$, an eigenvalue of $A^{\prime}$ and the corresponding eigenvector $\boldsymbol{v} \in \mathbb{C}^{d} \backslash\{0\}$. We get

$$
0 \leq \overline{\boldsymbol{v}}^{\prime}\left(\Gamma_{d}-A \Gamma_{d} A^{\prime}\right) \boldsymbol{v}=\overline{\boldsymbol{v}}^{\prime}\left(\Gamma_{d}-A \Gamma_{d} A^{\prime}\right) \boldsymbol{v}=\overline{\boldsymbol{v}}^{\prime} \Gamma_{d} \boldsymbol{v}\left(1-|\lambda|^{2}\right) .
$$

We conclude that $|\lambda| \leq 1$ since $\overline{\boldsymbol{v}}^{\prime} \Gamma_{d} \boldsymbol{v}>0$.

Lemma A.5. Let $d \geq 1,\left(X_{t, T}\right)_{t \in \mathbb{Z}, T \geq T_{0}}$ be an array process and $h:[0,1] \rightarrow \mathbb{R}$. For any $M \in \mathbb{N}^{*}$, define the local tapered empirical covariance function $\widehat{\gamma}_{T, M}$ as in Definition 2.8 and let, for any $t \in \mathbb{Z}$ and $T \geq T_{0}, \widehat{\Gamma}_{t, T, M}=\left(\widehat{\gamma}_{T, M}(t / T, i-j) ; i, j=\right.$ $1, \ldots, d)$ be the corresponding $d \times d$ empirical covariance matrix. Then, either $\widehat{\Gamma}_{t, T, M}$ is non-singular, or $\widehat{\gamma}_{T, M}(t / T, \ell)=0$ for all $\ell \in \mathbb{Z}$. Moreover, in the case where it is non-singular, the Yule-Walker estimate $\widehat{\boldsymbol{\theta}}_{t, T}(M)$ defined by (2.16) satisfies $\left\|\widehat{\boldsymbol{\theta}}_{t, T}(M)\right\| \leq 2^{d}-1$.

Proof: First note that for all $u \in \mathbb{R}$, the sequence $\left(\widehat{\gamma}_{T, M}(u, \ell)\right)_{\ell \in \mathbb{Z}}$ is the covariance function associated with the spectral density

$$
\widehat{f}_{M}(u, \lambda)=\frac{1}{H_{M}}\left|\sum_{t=1}^{M} h\left(\frac{t}{M}\right) X_{\lfloor u T\rfloor+t-M / 2, T} \mathrm{e}^{-\mathrm{i} \lambda t}\right|^{2}
$$

We conclude by applying Lemmas A.2 (i) and A.4.

\section{Appendix B. Bounds of the estimation risk in the general setting}

B.1. Proof of Lemma 2.7. Let us first bound the approximation error $\Gamma_{t / T}-\Gamma_{t, T}^{*}$.

$$
\begin{aligned}
\left|\gamma\left(\frac{t}{T}, i-j\right)-\gamma^{*}(t-i, T, j-i)\right| \leq\left|\gamma\left(\frac{t-i}{T}, j-i\right)-\gamma\left(\frac{t}{T}, i-j\right)\right| \\
+\left|\gamma\left(\frac{t-i}{T}, j-i\right)-\gamma^{*}(t-i, T, j-i)\right|
\end{aligned}
$$


The second line term of Inequality (B.1) is upper bounded by $R T^{-\min (1, \beta)}$ because of Inequality (2.3). Using the local covariance expression (2.2), Cauchy-Schwartz inequality and $f(, \lambda) \in \Lambda_{1}(\beta, R)$, the following holds for $T \geq d \geq|i|$,

$$
\begin{aligned}
\left|\gamma\left(\frac{t-i}{T}, j-i\right)-\gamma\left(\frac{t}{T}, i-j\right)\right| & =\left|\int_{-\pi}^{\pi} \mathrm{e}^{\mathrm{i}(j-i) \lambda}\left(f\left(\frac{t-i}{T}, \lambda\right)-f\left(\frac{t}{T}, \lambda\right)\right) \mathrm{d} \lambda\right| \\
& \leq 2 \pi d R T^{-\min (1, \beta)} .
\end{aligned}
$$

Inequality (2.3) implies that $\left\|\gamma_{t, T}^{*}-\gamma_{t / T}\right\| \leq d^{1 / 2} R T^{-\min (1, \beta)}$ and inequalities (B.1), (B.2) and again (2.3) imply that for $T \geq d$,

$$
\left\|\Gamma_{t / T}-\Gamma_{t, T}^{*}\right\| \leq d(2 \pi d R+R) T^{-\min (1, \beta)} .
$$

The smallest eigenvalue of the matrix $\Gamma_{t / T}$ is greater or equal to $2 \pi f_{-}$(see Lemma A.2 (ii)). Observe that

$$
\begin{aligned}
& \inf _{t} \inf _{\|\boldsymbol{a}\|=1} \boldsymbol{a}^{\prime} \Gamma_{t, T}^{*} \boldsymbol{a}=\inf _{t} \inf _{\|\boldsymbol{a}\|=1}\left\{\boldsymbol{a}^{\prime}\left(\Gamma_{t, T}^{*}-\Gamma_{t / T}\right) \boldsymbol{a}+\boldsymbol{a}^{\prime} \Gamma_{t / T} \boldsymbol{a}\right\} \\
& \geq \inf _{t} \inf _{\|\boldsymbol{a}\|=1} \boldsymbol{a}^{\prime}\left(\Gamma_{t, T}^{*}-\Gamma_{t / T}\right) \boldsymbol{a}+\inf _{t} \inf _{\|\boldsymbol{a}\|=1} \boldsymbol{a}^{\prime} \Gamma_{t / T} \boldsymbol{a} \geq 2 \pi f_{-}-d^{3 / 2} C T^{-\min (1, \beta)} .
\end{aligned}
$$

Then, for $T \geq T_{0}=\left(d R(2 \pi d+1) /\left(2 \pi f_{-}\right)\right)^{1 / \min (1, \beta)}$, we have that $\Gamma_{t, T}^{*}$ is invertible and $\left\|\left(\Gamma_{t, T}^{*}\right)^{-1}\right\| \leq\left(\pi f_{-}\right)^{-1}$. Now, from equations (2.11) and (2.12) we obtain that

$$
\boldsymbol{\theta}_{t, T}^{*}-\boldsymbol{\theta}_{t / T}=\left(\Gamma_{t, T}^{*}\right)^{-1}\left[\left(\Gamma_{t / T}-\Gamma_{t, T}^{*}\right) \boldsymbol{\theta}_{t / T}+\boldsymbol{\gamma}_{t, T}^{*}-\gamma_{t / T}\right] .
$$

Applying matrix inequalities (specifically with the spectral norm) we get

$$
\left\|\boldsymbol{\theta}_{t, T}^{*}-\boldsymbol{\theta}_{t / T}\right\| \leq\left\|\left(\Gamma_{t, T}^{*}\right)^{-1}\right\|\left(\left\|\Gamma_{t / T}-\Gamma_{t, T}^{*}\right\|\left\|\boldsymbol{\theta}_{t / T}\right\|+\left\|\gamma_{t, T}^{*}-\gamma_{t / T}\right\|\right) .
$$

Lemma A.4 ensures that $\left\|\boldsymbol{\theta}_{t / T}\right\| \leq 2^{d}$ and the result follows with $C_{1}=$ $\left(\pi f_{-}\right)^{-1}\left(d(2 \pi d R+R) 2^{d}+R\right)$.

B.2. Bias Approximation. The following elementary lemma will be useful.

Lemma B.1. Let $h:[0,1] \rightarrow \mathbb{R}$ satisfying $(\mathbf{H})$. Then, for all $\ell=0,1,2, \ldots$ and $M \geq j \geq 0$, we have

$$
\frac{1}{M} \sum_{s=j+1}^{M} h\left(\frac{s}{M}\right) h\left(\frac{s-j}{M}\right)\left(\frac{s}{M}\right)^{\ell}=\int_{0}^{1} h^{2}(u) u^{\ell} \mathrm{d} u+O_{j, \ell,\|h\|_{\infty},\left\|h^{\prime}\right\|_{\infty}}\left(M^{-1}\right) .
$$

In particular, in the case $j=\ell=0, H_{M}=M+O_{\|h\|_{\infty},\left\|h^{\prime}\right\|_{\infty}}(1)$.

Proof: The proof is straightforward using, for $M$ large enough, Riemann approximations on the blocks defined by $s / M \in\left(u_{i-1}+j / M, u_{i}\right]$, for $i=1, \ldots, N$, and neglecting the terms from the indices $s$ such that $s / M \in\left(u_{i-1}, u_{i-1}+j / M\right]$, the number of which is bounded.

We can now derive the following approximation of the bias.

Theorem B.2. Suppose that Assumption (M-1) holds with some $\beta>0$ and $R>0$, and let $h:[0,1] \rightarrow \mathbb{R}$ satisfying $(\mathbf{H})$. Let $k \in \mathbb{N}$ and $\alpha \in(0,1]$ be uniquely defined 
such that $\beta=k+\alpha$. Then, for all $j \in \mathbb{Z}$ and $M \in 2 \mathbb{N}^{*}$, we have

$$
\begin{aligned}
\mathbb{E}\left[\widehat{\gamma}_{T, M}\left(\frac{t}{T}, j\right)\right]= & \gamma\left(\frac{t}{T}, j\right)+\sum_{\ell=1}^{k} c_{h, f, j, \ell}\left(\frac{M}{T}\right)^{\ell} \\
& +O_{j,\|h\|_{\infty},\left\|h^{\prime}\right\|_{\infty}, \beta, R}\left(\frac{1}{M}+\left(\frac{M}{T}\right)^{\beta}\right),
\end{aligned}
$$

where $c_{h, f, j, \ell} \in \mathbb{C}$ only depends on $h$, the spectral density $f, j$ and $\ell$. If $h(x)=$ $h(1-x)$ for all $x \in[0,1]$, then $c_{h, f, j, 1}=0$.

Proof: Without loss of generality we let moreover assume $M \geq j \geq 0$, in which case

$$
\mathbb{E}\left[\widehat{\gamma}_{T, M}\left(\frac{t}{T}, j\right)\right]=\frac{1}{H_{M}} \sum_{s=j+1}^{M} h\left(\frac{s}{M}\right) h\left(\frac{s-j}{M}\right) \gamma^{*}\left(t+s-\frac{M}{2}, T, j\right) .
$$

Since $|h|$ is also piecewise continuously differentiable, Lemma B.1 gives that

$$
\frac{1}{\left|H_{M}\right|} \sum_{s=j+1}^{M}\left|h\left(\frac{s}{M}\right) h\left(\frac{s-j}{M}\right)\right|=O_{j,\|h\|_{\infty},\left\|h^{\prime}\right\|_{\infty}}(1) .
$$

With Inequality (2.3), we obtain that

$$
\begin{aligned}
\mathbb{E}\left[\widehat{\gamma}_{T, M}\left(\frac{t}{T}, j\right)\right]=\gamma_{M, j}+O_{j,\|h\|_{\infty},\left\|h^{\prime}\right\|_{\infty}, R}\left(T^{-\min (1, \beta)}\right) \\
\text { where } \gamma_{M, j}:=\frac{1}{H_{M}} \sum_{s=j+1}^{M} h\left(\frac{s}{M}\right) h\left(\frac{s-j}{M}\right) \gamma\left(\frac{t+s-M / 2}{T}, j\right) .
\end{aligned}
$$

Since $f(\cdot, \lambda) \in \Lambda_{1}(\beta, R)$, a Taylor expansion yields

$$
f\left(\frac{t-M / 2+s}{T}, \lambda\right)=\sum_{\ell=0}^{k} \frac{\partial_{1}^{\ell} f(t / T, \lambda)}{\ell !}\left(\frac{-M / 2+s}{T}\right)^{\ell}+f_{k}\left(\frac{t}{T}, \frac{-M / 2+s}{T}, \lambda\right)
$$

with

$$
\sup _{t \in \mathbb{Z}, 1 \leq s \leq M}\left|f_{k}(t / T,(-M / 2+s) / T, \lambda)\right|=O_{\beta, R}\left((M / T)^{\beta}\right) .
$$

Then

$$
\begin{aligned}
& \gamma_{M, j}=\frac{1}{H_{M}} \int_{-\pi}^{\pi} \mathrm{e}^{\mathrm{i} j \lambda} \sum_{s=j+1}^{M} h\left(\frac{s}{M}\right) h\left(\frac{s-j}{M}\right) f\left(\frac{t-M / 2+s}{T}, \lambda\right) \mathrm{d} \lambda= \\
& \sum_{\ell=0}^{k} \int_{-\pi}^{\pi} \frac{\partial_{1}^{\ell} f(t / T, \lambda)}{\ell !} \mathrm{e}^{\mathrm{i} j \lambda} \frac{1}{H_{M}} \sum_{s=j+1}^{M} h\left(\frac{s}{M}\right) h\left(\frac{s-j}{M}\right)\left(\frac{-M / 2+s}{T}\right)^{\ell} \mathrm{d} \lambda \\
& \quad+\int_{-\pi}^{\pi} \mathrm{e}^{\mathrm{i} j \lambda} \frac{1}{H_{M}} \sum_{s=j+1}^{M} h\left(\frac{s}{M}\right) h\left(\frac{s-j}{M}\right) f_{k}\left(\frac{t}{T}, \frac{-M / 2+s}{T}, \lambda\right) \mathrm{d} \lambda .
\end{aligned}
$$


Lemma B.1 yields that for all $\ell=1, \ldots, k$,

$$
\begin{aligned}
& \frac{1}{H_{M}} \sum_{s=j+1}^{M} h\left(\frac{s}{M}\right) h\left(\frac{s-j}{M}\right)\left(\frac{-M / 2+s}{T}\right)^{\ell} \\
& =\left(\frac{M}{T}\right)^{\ell} \frac{M}{H_{M}} \frac{1}{M} \sum_{s=j+1}^{M} h\left(\frac{s}{M}\right) h\left(\frac{s-j}{M}\right)\left(-\frac{1}{2}+\frac{s}{M}\right)^{\ell} \\
& =c_{h, \ell}\left(\frac{M}{T}\right)^{\ell}+O_{j,\|h\|_{\infty},\left\|h^{\prime}\right\|_{\infty}, \ell}\left(\frac{1}{M}\right)\left(\frac{M}{T}\right)^{\ell},
\end{aligned}
$$

with $c_{h, \ell}=\int_{0}^{1} h^{2}(u)\left(u-\frac{1}{2}\right)^{\ell} \mathrm{d} u$. Observe that $c_{h, 0}=1$ by assumption in $(\mathbf{H})$, and, if $h(x)=h(1-x)$ for all $x \in[0,1]$, then moreover $c_{h, 1}=0$. From this it follows that

$$
\begin{aligned}
& \int_{-\pi}^{\pi} \frac{\partial_{1}^{\ell} f(t / T, \lambda)}{\ell !} \frac{\mathrm{e}^{\mathrm{i} j \lambda}}{H_{M}} \sum_{s=j+1}^{M} h\left(\frac{s}{M}\right) h\left(\frac{s-j}{M}\right)\left(\frac{-M / 2+s}{T}\right)^{\ell} \mathrm{d} \lambda \\
& =\left(\frac{M}{T}\right)^{\ell}\left(c_{h, f, j, \ell}+O_{j,\|h\|_{\infty},\left\|h^{\prime}\right\|_{\infty}, \beta, R}\left(\frac{1}{M}\right)\right),
\end{aligned}
$$

where, in particular, $c_{h, f, j, 0}=\gamma(t / T, j)$ and $c_{h, f, j, 1}=0$ if $h(x)=h(1-x)$. Finally, by (B.4), and since $|h|$ is also piecewise continuously differentiable, the reminder term in (B.5) satisfies

$$
\begin{aligned}
& \int_{-\pi}^{\pi} \mathrm{e}^{\mathrm{i} j \lambda} \frac{1}{H_{M}} \sum_{s=j+1}^{M} h\left(\frac{s}{M}\right) h\left(\frac{s-j}{M}\right) f_{k}\left(\frac{t-M / 2+s}{T}, \lambda\right) \mathrm{d} \lambda \\
& =O_{j,\|h\|_{\infty},\left\|h^{\prime}\right\|_{\infty}, \beta, R}\left(\left(\frac{M}{T}\right)^{\beta}\right) .
\end{aligned}
$$

We thus finally obtain that

$$
\gamma_{M, j}=\gamma\left(\frac{t}{T}, j\right)+\sum_{\ell=1}^{k} c_{h, f, j, \ell}\left(\frac{M}{T}\right)^{\ell}+O_{d,\|h\|_{\infty},\left\|h^{\prime}\right\|_{\infty}, \beta, R}\left(\frac{1}{M}+\left(\frac{M}{T}\right)^{\beta}\right)
$$

This approximation and the bound (B.3) allow us to conclude the proof.

B.3. Proof of Theorem 3.2. Gathering together Assumption (C) and Theorem B.2 yields, for any $j=-d, \ldots, d$,

$$
\begin{aligned}
\widehat{\gamma}_{T, M}\left(\frac{t}{T}, j\right)=\gamma\left(\frac{t}{T}, j\right)+\sum_{\ell=1}^{k} c_{h, f, j, \ell}\left(\frac{M}{T}\right)^{\ell} \\
+\quad O_{d,\|h\|_{\infty},\left\|h^{\prime}\right\|_{\infty}, \beta, R}\left(\frac{1}{M}+\left(\frac{M}{T}\right)^{\beta}\right)+u_{M}\left(\frac{t}{T}, j\right),
\end{aligned}
$$

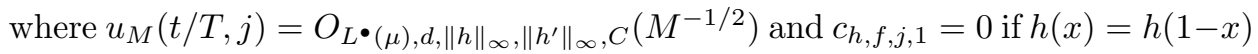
for all $x \in[0,1]$. 
For the sake of simplicity, we drop $t, T$ in the notation and set $\gamma=\gamma_{t / T}, \widehat{\gamma}_{M}=$ $\widehat{\gamma}_{t, T, M}, \Gamma:=\Gamma_{t / T}$ and $\widehat{\Gamma}_{M}:=\widehat{\Gamma}_{t, T, M}$. Using the expression (B.6), we obtain

$$
\begin{aligned}
& \Gamma-\widehat{\Gamma}_{M}=\sum_{\ell=1}^{k} C_{h, f, \ell}\left(\frac{M}{T}\right)^{\ell}+O_{d,\|h\|_{\infty},\left\|h^{\prime}\right\|_{\infty}, \beta, R}\left(\frac{1}{M}+\left(\frac{M}{T}\right)^{\beta}\right)+U_{M}, \\
& \widehat{\gamma}_{M}-\gamma=\sum_{\ell=1}^{k} c_{h, f, \ell}\left(\frac{M}{T}\right)^{\ell}+O_{d,\|h\|_{\infty},\left\|h^{\prime}\right\|_{\infty}, \beta, R}\left(\frac{1}{M}+\left(\frac{M}{T}\right)^{\beta}\right)+\boldsymbol{u}_{M}
\end{aligned}
$$

where the matrices $C_{h, f, \ell} \in \mathbb{R}^{d \times d}$ and the vectors $\boldsymbol{c}_{h, f, \ell} \in \mathbb{R}^{d}$ only depend on $d, h, f$ and $\ell$. Furthermore $U_{M}$ and $\boldsymbol{u}_{M}$ both are $O_{L} \bullet(\mu), d,\|h\|_{\infty},\left\|h^{\prime}\right\|_{\infty}, C\left(M^{-1 / 2}\right)$. Again $C_{h, f, 1}=0$ and $\boldsymbol{c}_{h, f, 1}=0$ if $h(x)=h(1-x)$ for all $x \in[0,1]$.

Note now that Lemma A.2 with the assumption $f_{-}>0$ further says that $\Gamma_{t / T}$ is also non-singular with $\Gamma_{t / T}^{-1}=O_{f_{-}}(1)$ and, with (2.17), we can thus apply Lemma A.1, showing that (A.3) holds with $\boldsymbol{\theta}=\boldsymbol{\theta}_{t / T}, \widehat{\boldsymbol{\theta}}=\widehat{\boldsymbol{\theta}}_{t, T}(M), \boldsymbol{\gamma}=\boldsymbol{\gamma}_{t / T}$, $\widehat{\gamma}=\widehat{\gamma}_{M}=\widehat{\gamma}_{t, T, M}, \Gamma:=\Gamma_{t / T}$ and $\widehat{\Gamma}=\widehat{\Gamma}_{M}:=\widehat{\Gamma}_{t, T, M}$. Next the bounds provided by Lemmas A.4 and (2.17) further imply $\|\boldsymbol{\theta}\|$ and $\|\widehat{\boldsymbol{\theta}}\|$ to be less that $2^{d}$. This, with the approximations (B.7) and (B.8), yields (3.4).

B.4. Proof of Theorem 3.4. For each $j=0, \ldots, k$ replace $M$ by $M / 2^{j}$ in (3.4), multiply the resulting expression by $\omega_{j}$ and sum over $j$. Matrix $A$ (definition below Equation (3.5)) is a non singular Vandermonde matrix and $\boldsymbol{\omega}$ is well defined.

\section{Appendix C. Postponed proofs for TVCBS and TVAR processes}

C.1. Proof of Theorem 3.1. Let us denote, for any $L^{p}$ random variable $Z,\|Z\|_{p}=$ $\left(\mathbb{E}\left[|Z|^{p}\right]\right)^{1 / p}$ its $L^{p}$-norm. The proof relies on the Burkhölder inequality for nonstationary dependent sequences. Namely, an immediate consequence of Dedecker et al. (2007, Proposition 5.4) and the Hölder and Minkowskii inequalities is that if $p \geq 2$ and $\left(Z_{t}\right)_{t \in \mathbb{Z}}$ is a $L^{p}$ process adapted to the filtration $\left(\mathcal{F}_{t}\right)_{t \in \mathbb{Z}}$, then, for all $s \in \mathbb{Z}$ and $n \geq 1$

$$
\begin{aligned}
& \left\|\sum_{t=s+1}^{s+n}\left(Z_{t}-\mathbb{E}\left[Z_{t}\right]\right)\right\|_{p}^{2} \\
& \leq 2 p n\left(\sup _{t \in \mathbb{Z}}\left\|Z_{t}-\mathbb{E}\left[Z_{t}\right]\right\|_{p}\right)\left(\sup _{t \in \mathbb{Z}} \sum_{k=0}^{\infty}\left\|\mathbb{E}\left[Z_{t+k} \mid \mathcal{F}_{t}\right]-\mathbb{E}\left[Z_{t+k}\right]\right\|_{p}\right) .
\end{aligned}
$$

Applying this inequality with $s=|\ell|+1, n=M-|\ell|$ to

$$
Z_{t}=h\left(\frac{t}{M}\right) h\left(\frac{t-|\ell|}{M}\right) X_{\lfloor u T\rfloor+t-M / 2, T} X_{\lfloor u T\rfloor+t-|\ell|-M / 2, T}, \quad \mathcal{F}_{t}=\mathcal{F}_{\lfloor u T\rfloor+t-M / 2}^{\xi},
$$


where $\mathcal{F}_{t}^{\xi}=\sigma\left(\xi_{s}, s \leq t\right)$ denotes the natural filtration of $\left(\xi_{t}\right)_{t \in \mathbb{Z}}$, we obtain that, for any $M>|\ell|$,

$$
\begin{aligned}
& \left\|\widehat{\gamma}_{T, M}(u, \ell)-\mathbb{E}\left[\widehat{\gamma}_{T, M}(u, \ell)\right]\right\|_{p} \leq \frac{2(p(M-|\ell|))^{1 / 2}}{H_{M}}\|h\|_{\infty}^{2} \sup _{s \in \mathbb{Z}}\left\|X_{s, T}\right\|_{2 p} \\
& \times\left(\sup _{t \in \mathbb{Z}} \sum_{k=0}^{\infty}\left\|\mathbb{E}\left[X_{t+k, T} X_{t-|\ell|+k, T} \mid \mathcal{F}_{t}^{\xi}\right]-\mathbb{E}\left[X_{t+k, T} X_{t-|\ell|+k, T}\right]\right\|_{p}\right)^{1 / 2} .
\end{aligned}
$$

Under Assumption (H), $H_{M} / M \in(1 / 2,3 / 2)$ for $M$ large enough (see Lemma B.1) and it is thus now sufficient to show that, for any $p \geq 2$,

$$
\begin{aligned}
& \sup _{s \in \mathbb{Z}}\left\|X_{s, T}\right\|_{2 p}=O_{K, r,\left(\psi_{k}\right)}(1) \\
& \sup _{t \in \mathbb{Z}} \sum_{k=0}^{\infty}\left\|\mathbb{E}\left[X_{t+k, T} X_{t-|\ell|+k, T} \mid \mathcal{F}_{t}^{\xi}\right]-\mathbb{E}\left[X_{t+k, T} X_{t-|\ell|+k, T}\right]\right\|_{p}=O_{K, r, k_{0}, \ell,\left(\psi_{k}\right),\left(\zeta_{k}\right)}(1)
\end{aligned}
$$

The bound (C.1) is a direct consequence of (2.6) and (2.7) and the assumption on

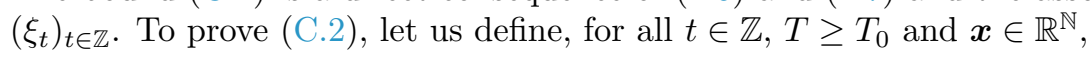

$$
\Phi_{t, T}^{0}(\boldsymbol{x})=\varphi_{t, T}^{0}(\boldsymbol{x}) \varphi_{t-|\ell|, T}^{0}\left(\left(x_{j+|\ell|}\right)_{j \geq 0}\right) .
$$

It then follows that $X_{t, T} X_{t-|\ell|, T}=\Phi_{t, T}^{0}\left(\left(\xi_{t-j}\right)_{j \geq 0}\right)$ and is then straightforward to show that the assumptions on $\varphi^{0}$ yields that there exists some constant $K^{\prime}$ only depending on $K$ and $\left(\psi_{j}\right)_{j \in \mathbb{N}}$ and $\left(\zeta_{j}\right)_{j \in \mathbb{N}}$ such that, for all $t \in \mathbb{Z}, T \geq T_{0}$ and all $\boldsymbol{x}, \boldsymbol{x}^{\prime} \in \mathbb{R}^{\mathbb{N}}$ satisfying $x_{j}=x_{j}^{\prime}$ for $1 \leq j \leq k_{0}+|\ell|$,

$$
\begin{aligned}
& \left|\Phi_{t, T}^{0}(\boldsymbol{x})\right| \leq K^{\prime}\left(1+\sum_{j=0}^{\infty} \tilde{\psi}_{j}\left|x_{j}\right|\right)^{2 r}, \\
& \left|\Phi_{t, T}^{0}(\boldsymbol{x})-\Phi_{t, T}^{0}\left(\boldsymbol{x}^{\prime}\right)\right| \leq K^{\prime}\left(\sum_{j \geq 0} \tilde{\zeta}_{j}\left|x_{k_{0}+j}-x_{k_{0}+j}^{\prime}\right|\right)\left(1+\sum_{j \geq 0} \tilde{\psi}_{j}\left(\left|x_{j}\right|+\left|x_{j}^{\prime}\right|\right)\right)^{2 r-1},
\end{aligned}
$$

where $\tilde{\psi}_{j}=\psi_{j}$ and $\tilde{\zeta}_{j}=\zeta_{j}$ for $0 \leq j<|\ell|$, and $\tilde{\psi}_{j}=\max \left(\psi_{j-|\ell|}, \psi_{j}\right)$ and $\tilde{\zeta}_{j}=$ $\zeta_{j-|\ell|}+\zeta_{j}$ for $j \geq|\ell|$. By Jensen's inequality, we have that, for all $k \geq 0$,

$$
\begin{aligned}
& \left\|\mathbb{E}\left[X_{t+k, T} X_{t-|\ell|+k, T} \mid \mathcal{F}_{t}^{\xi}\right]-\mathbb{E}\left[X_{t+k, T} X_{t-|\ell|+k, T}\right]\right\|_{p} \\
& \leq\left\|\Phi_{t+k, T}^{0}\left(\left(\xi_{t+k-j}\right)_{j \geq 0}\right)-\Phi_{t+k, T}^{0}\left(\left(\xi_{t+k-j}^{\prime}\right)_{j \geq 0}\right)\right\|_{p},
\end{aligned}
$$

where $\left(\xi_{s}^{\prime}\right)_{s \in \mathbb{Z}}$ is i.i.d. with same distribution as $\left(\xi_{s}\right)_{s \in \mathbb{Z}}$ and such that $\xi_{t+k-j}=$ $\xi_{t+k-j}^{\prime}$ for all $j=0,1, \ldots, k-1$ and $\left(\xi_{t-j}^{\prime}\right)_{j \geq 0}$ and $\left(\xi_{t-j}\right)_{j \geq 0}$ are independent. With the above bounds on $\Phi^{0}$ and using the Minkowskii and Hölder inequalities, we thus get that, for all $t \in \mathbb{Z}$ and $k \geq 0$,

$$
\left\|\mathbb{E}\left[X_{t+k, T} X_{t-|\ell|+k, T} \mid \mathcal{F}_{t}^{\xi}\right]-\mathbb{E}\left[X_{t+k, T} X_{t-|\ell|+k, T}\right]\right\|_{p} \leq 2 K^{\prime}\left(1+\|\tilde{\boldsymbol{\psi}}\|_{1}\left\|\xi_{0}\right\|_{2 r p}\right)^{2 r},
$$

and, if $k>k_{0}+|\ell|$, the same quantity is bounded from above by

$$
2 K^{\prime}\left\|\xi_{0}\right\|_{2 p}\left(1+2\|\tilde{\boldsymbol{\psi}}\|_{1}\left\|\xi_{0}\right\|_{2 p(2 r-1)}\right)^{2 r-1}\left(\sum_{j=k-k_{0}}^{\infty} \tilde{\zeta}_{j}\right) .
$$


Summing these bounds over all $k \geq 0$, we obtain

$$
\begin{gathered}
\sum_{k=0}^{\infty}\left\|\mathbb{E}\left[X_{t+k, T} X_{t-|\ell|+k, T} \mid \mathcal{F}_{t}^{\xi}\right]-\mathbb{E}\left[X_{t+k, T} X_{t-|\ell|+k, T}\right]\right\|_{p} \\
\leq 2 K^{\prime}\left(1+2\|\tilde{\boldsymbol{\psi}}\|_{1}\left\|\xi_{0}\right\|_{4 r p}\right)^{2 r}\left(k_{0}+|\ell|+\sum_{k>k_{0}+|\ell|} \sum_{j=k-k_{0}}^{\infty} \tilde{\zeta}_{j}\right) \\
=2 K^{\prime}\left(1+2\|\tilde{\boldsymbol{\psi}}\|_{1}\left\|\xi_{0}\right\|_{4 r p}\right)^{2 r}\left(k_{0}+|\ell|+\sum_{j>|\ell|}(j-|\ell|) \tilde{\zeta}_{j}\right) .
\end{gathered}
$$

By (3.3), the term between parentheses is a finite constant only depending on $k_{0}$, $|\ell|$ and $\boldsymbol{\zeta}$. We thus have shown (C.2), which concludes the proof.

C.2. Proof of Theorem 4.1. We first need to recall some basic facts on the representation of a TVAR process as a TVCBS. Let us set $\boldsymbol{e}_{1}=\left[\begin{array}{lll}1 & 0 & \ldots\end{array}\right]^{\prime} \in \mathbb{R}^{p}$ and introduce the companion $p \times p$ matrices defined for all $u \in \mathbb{R}$ by

$$
A(u)=\left[\begin{array}{ccccc}
\theta_{1}(u) & \theta_{2}(u) & \ldots & \ldots & \theta_{p}(u) \\
1 & 0 & \ldots & \ldots & 0 \\
0 & 1 & 0 & \ldots & 0 \\
\vdots & \ddots & \ddots & \ddots & \vdots \\
0 & \ldots & 0 & 1 & 0
\end{array}\right] .
$$

By Giraud et al. (2015, Proposition 1 and its proof), the TVAR process $\left(X_{t, T}\right)_{t \in \mathbb{Z}, T \geq T_{0}}$ is a special case of TVCBS introduced in Example 2.5. Namely, it satisfies a representation (2.6) with a linear form

$$
\varphi_{t, T}^{0}(\boldsymbol{x})=\sum_{j=0}^{\infty} a_{t, T}(j) \sigma((t-j) / T) x_{j}, \quad \text { with } \quad a_{t, T}(j)=\boldsymbol{e}_{1}^{\prime}\left[\prod_{i=1}^{j} A\left(\frac{t-i}{T}\right)\right] \boldsymbol{e}_{1},
$$

and moreover, there exist some constants $\bar{K}>0$ and $\delta_{1} \in(0,1)$ only depending on $p, \delta, \beta$ and $R_{0}$ such that, for all $T \geq T_{0}, t \in \mathbb{Z}$ and $j \in \mathbb{N}^{*}$,

$$
\left\|\prod_{i=1}^{j} A\left(\frac{t-i}{T}\right)\right\| \leq \bar{K} \delta_{1}^{j}
$$

Hence in particular

$$
\left|a_{t, T}(j)\right| \leq \bar{K} \delta_{1}^{j}
$$

implying that $\varphi^{0}$ above satisfies (2.7) and (3.2) with $r=1, K=1$ and $\psi_{j}=\zeta_{j}=$ $\bar{K} \sigma_{+} \delta_{1}^{j}$.

We can now proceed with the proof of Theorem 4.1, starting with (i). To show that it is weakly locally stationary with the local spectral density of the $\operatorname{AR}(p)$ with local standard deviation $\sigma(u)$ and autoregresive coefficients $\theta_{1}(u), \ldots, \theta_{p}(u)$, it only remains to show that (2.8) holds with $r=1$ and

$$
\varphi(u, \boldsymbol{x})=\sigma(u) \sum_{j=0}^{\infty} \boldsymbol{e}_{1}^{\prime} A^{j}(u) \boldsymbol{e}_{1} x_{j}
$$


This is a simple consequence of (C.3) and the fact that $\sigma \in \Lambda_{1}\left(\min (1, \beta), R_{0}\right)$ and $\boldsymbol{\theta} \in \Lambda_{p}\left(\min (1, \beta), R_{0}\right)$ by assumption. Then (4.4) follows from the representation (2.6) : using that it is causal and (2.9), we get in the case $d=p$ that $\boldsymbol{\theta}(t / T)=\boldsymbol{\theta}_{t, T}^{*}$. On the other hand, the mere definition of the local spectral density in (4.3) (being that of an $\operatorname{AR}(p)$ process at fixed $u$ ), the case $d=p$ yields that $\boldsymbol{\theta}_{u}=\boldsymbol{\theta}(u)$. Finally, an additional consequence of the definition of $f$ is that the spectral density $f(\cdot, \lambda)$ belongs to $\Lambda_{1}(\beta, R)$ for any $\lambda \in \mathbb{R}$ with $R$ only depending on $p, \delta, \beta, \sigma_{+}$and $R_{0}$, provided that we can show that $\left|\boldsymbol{\theta}\left(\mathrm{e}^{-\mathrm{i} \lambda} ; u\right)\right|$ can be bounded from below by a positive constant only depending on $\delta$ and $p$. By Lemma A.3 and continuity of $(\lambda, \boldsymbol{\theta}) \mapsto 1-\sum_{j=1}^{p} \theta_{j} \mathrm{e}^{-\mathrm{i} j \lambda}$, and since $\delta^{-1}>1$ we have that

$$
0<\inf _{\boldsymbol{\theta} \in s_{(p)}(\delta), \lambda \in \mathbb{R}}\left|1-\sum_{j=1}^{p} \theta_{j} \mathrm{e}^{-\mathrm{i} j \lambda}\right| \leq \sup _{\boldsymbol{\theta} \in s_{(p)}(\delta), \lambda \in \mathbb{R}}\left|1-\sum_{j=1}^{p} \theta_{j} \mathrm{e}^{-\mathrm{i} j \lambda}\right|<\infty .
$$

Of course these two constants only depend on $\delta$ and $p$ and the inf one can serve as a lower bound of $\left|\boldsymbol{\theta}\left(\mathrm{e}^{-\mathrm{i} \lambda} ; u\right)\right|$, concluding the proof of (i).

Next, we prove (ii) and (iii), which respectively require the two add-on properties

(a) $f(u, \lambda) \geq f_{-}$for all $u, \lambda \in \mathbb{R}$,

(b) if $\xi_{0}$ has a diffuse distribution, then $\mathbb{P}\left(X_{t, T}=0\right)=0$ for all $t \in \mathbb{Z}$ and $T \geq T_{0}$.

The fact (a) follows from (4.3), $\sigma(u) \geq \rho \sigma_{+}$and the upper bounds in (C.4), which shows that we can find such an $f_{-}>0$ only depending on $p, \delta, \rho$ and $\sigma_{+}$. Fact (b) is a consequence of the TVCBS representation above and the assumptions on $\left(\xi_{t}\right)$ which implies that for all $t, T$ and $j \in \mathbb{N}$, we can write $X_{t, T}=a_{t, T}(j) \xi_{t-j}+Z_{t, T}(j)$ with $Z_{t, T}(j)$ independent of $\xi_{t-j}$. Hence, if $\xi_{0}$ has a diffuse distribution, it only remains to prove that for all $T \geq T_{0}$ and all $t \in \mathbb{Z}$, there exists $j \in \mathbb{N}$ such that $a_{t, T}(j) \neq 0$. Using again the TVCBS representation above, this is equivalent to show that for all $T \geq T_{0}$ and all $t \in \mathbb{Z}, \gamma^{*}(t, T, 0)=\operatorname{var}\left(X_{t, T}\right)>0$. Now observe that by (a) and since $\left(X_{t, T}\right)_{t \in \mathbb{Z}, T \geq T_{0}}$ is $(\beta, R)$-weakly locally stationary, we have

$$
\gamma^{*}(t, T, 0) \geq \gamma(t / T, 0)-R T^{-\min (1, \beta)} \geq 2 \pi f_{-}-R T^{-\min (1, \beta)}>0,
$$

where the last inequality holds by taking $T_{0}$ large enough (only depending on $f_{-}$ and $R$ and thus on $p, \delta, \rho, \sigma_{+}$and $R_{0}$.).

We conclude with the proof of (iv). We use the above TVCBS representation which were mentioned to satisfy (3.2) with $\zeta_{j}=\bar{K} \sigma_{+} \delta_{1}^{j}$ for some $\delta_{1}<1$. Hence (3.3) holds as well and we can apply Theorem 3.1, which shows that the TVAR process satisfies (C).

\section{References}

J. Baranger and C. Brezinski. Analyse numérique, volume 38. Hermann, Paris (1991). Collection Méthodes.

Andrew R. Barron. Are bayes rules consistent in information? In Thomas M. Cover and B. Gopinath, editors, Open Problems in Communication and Computation, pages 85-91. Springer New York (1987). ISBN 978-1-4612-9162-6. DOI: 10.1007/978-1-4612-4808-8_22.

P. J. Brockwell and R. A. Davis. Introduction to time series and forecasting. Springer Texts in Statistics. Springer-Verlag, New York, second edition (2002). ISBN 0-387-95351-5. MR1894099. 
P. J. Brockwell and R. A. Davis. Time series: theory and methods. Springer Series in Statistics. Springer, New York (2006). ISBN 978-1-4419-0319-8; 1-4419-0319-8. MR2839251.

N. Cesa-Bianchi and G. Lugosi. Prediction, learning, and games. Cambridge University Press, Cambridge (2006). ISBN 978-0-521-84108-5; 0-521-84108-9. MR2409394.

J. B. Conway. Functions of one complex variable. Springer-Verlag, New YorkHeidelberg (1973). Graduate Texts in Mathematics, 11.

R. Dahlhaus. Maximum likelihood estimation and model selection for locally stationary processes. J. Nonparametr. Statist. 6 (2-3), 171-191 (1996a). MR1383050.

R. Dahlhaus. On the Kullback-Leibler information divergence of locally stationary processes. Stochastic Process. Appl. 62 (1), 139-168 (1996b). MR1388767.

R. Dahlhaus and L. Giraitis. On the optimal segment length for parameter estimates for locally stationary time series. J. Time Ser. Anal. 19 (6), 629-655 (1998). MR1665941.

J. Dedecker, P. Doukhan, G. Lang, J. R. León R., S. Louhichi and C. Prieur. Weak dependence: with examples and applications, volume 190 of Lecture Notes in Statistics. Springer, New York (2007). ISBN 978-0-387-69951-6. MR2338725.

M. Duflo. Random iterative models, volume 34 of Applications of Mathematics (New York). Springer-Verlag, Berlin (1997). ISBN 3-540-57100-0. MR1485774.

T. W. Gamelin. Complex analysis. Undergraduate Texts in Mathematics. SpringerVerlag, New York (2001). ISBN 0-387-95093-1; 0-387-95069-9. MR1830078.

C. Giraud, F. Roueff and A. Sánchez-Pérez. Aggregation of predictors for nonstationary sub-linear processes and online adaptive forecasting of time varying autoregressive processes. Ann. Statist. 43 (6), 2412-2450 (2015). MR3405599.

U. Grenander and G. Szegő. Toeplitz forms and their applications. Chelsea Publishing Co., New York, second edition (1984). ISBN 0-8284-0321-X. MR890515.

T. Kley, P. Preuß and P. Fryzlewicz. Predictive, finite-sample model choice for time series under stationarity and non-stationarity. ArXiv Mathematics e-prints (2016). arXiv: 1611.04460.

H. R. Künsch. A note on causal solutions for locally stationary ar-processes (1995). Preprint ETH Zürich.

E. Moulines, P. Priouret and F. Roueff. On recursive estimation for time varying autoregressive processes. Ann. Statist. 33 (6), 2610-2654 (2005). MR2253097.

M. B. Priestley. Evolutionary spectra and non-stationary processes. (With discussion). J. Roy. Statist. Soc. Ser. B 27, 204-237 (1965). MR0199886.

T. Subba Rao, Suhasini Subba R. and C. R. Rao, editors. Time series analysis: methods and applications, volume 30 of Handbook of Statistics. Elsevier/NorthHolland, Amsterdam (2012). ISBN 978-0-444-53858-1. MR3295420.

P. Whittle. On the fitting of multivariate autoregressions, and the approximate canonical factorization of a spectral density matrix. Biometrika 50, 129-134 (1963). MR0161430. 